\title{
Picturing the elephant: Giant piezoelectric activity and the monoclinic phases of relaxor-ferroelectric single crystals
}

\author{
Matthew Davis
}

Received: 29 August 2006 / Accepted: 27 November 2006 / Published online: 21 February 2007

(C) Springer Science + Business Media, LLC 2007

\begin{abstract}
Understanding the relationship between the structure and "giant" piezoelectric properties of relaxor ferroelectric solid solutions $(1-x) \mathrm{Pb}\left(\mathrm{Mg}_{1 / 3} \mathrm{Nb}_{2 / 3}\right) \mathrm{O}_{3}-x \mathrm{PbTiO}_{3}[\mathrm{PMN}-$ $x \mathrm{PT}]$ and $(1-x) \mathrm{Pb}\left(\mathrm{Zn}_{1 / 3} \mathrm{Nb}_{2 / 3}\right) \mathrm{O}_{3}-x \mathrm{PbTiO}_{3}[\mathrm{PZN}-x \mathrm{PT}]$ is an extremely difficult task. In this article, three main paradigms are reviewed. In the first, the monoclinic phases present at the morphotropic phase boundary (MPB) are responsible for the giant piezoelectric response in that they allow, or at least facilitate, polarization rotation. In the second, a strong polarization rotation effect is explained by the large piezoelectric shear coefficients of zero-field rhombohedral and orthorhombic phases due to the near degeneracy at the MPB and the intrinsic softness of the relaxor state; zero-field monoclinic symmetries are explained by residually distorted rhombohedral and orthorhombic phases in the presence of internal stresses and/or residual bias fields. In the third, the monoclinic "phases" are composed of very finely twinned rhombohedral or tetragonal domains. In this "adaptive phase" model, based on that for ferroelastic martensites, the large electric-field induced strains are extrinsic in nature and result from the progressive switching of the component "nano-twins"; the ease of polarization rotation is explained by a high domain wall mobility. These paradigms remain to be mutually reconciled. The article includes a thorough review of the history of PMN$x \mathrm{PT}$ and $\mathrm{PZN}-x \mathrm{PT}$ single crystals and, particularly, the most important work done over the last decade.
\end{abstract}

Keywords Piezoelectric · Ferroelectric $\cdot$ Relaxor . Single crystal

\footnotetext{
M. Davis $(\bowtie)$

Laboratory of Ceramics, Ecole Polytechnique Fédérale de Lausanne (EPFL), Lausanne CH-1015, Switzerland

e-mail: matthew.davis@epfl.ch
}

\section{Introduction}

Relaxor-ferroelectric single crystals $(1-x) \mathrm{Pb}\left(\mathrm{Mg}_{1 / 3} \mathrm{Nb}_{2 / 3}\right)$ $\mathrm{O}_{3}-x \mathrm{PbTiO}_{3}[\mathrm{PMN}-x \mathrm{PT}]$ and $(1-x) \mathrm{Pb}\left(\mathrm{Zn}_{1 / 3} \mathrm{Nb}_{2 / 3}\right) \mathrm{O}_{3}-$ $x \mathrm{PbTiO}_{3}[\mathrm{PZN}-x \mathrm{PT}]$ have attracted a huge amount of attention over the last decade. When cut and poled along a non-polar, pseudocubic $\langle 001\rangle_{\mathrm{C}}$ direction rhombohedral, orthorhombic or monoclinic crystals exhibit "giant" piezoelectric coefficients $d_{33}>2,000 \mathrm{pm} / \mathrm{V}$ and high electromechanical coupling factors $k_{33}>0.90$. Such properties $[1,2]$ make them very attractive for next generation sensors and actuators and much work is currently being done to implement $\mathrm{PMN}-x \mathrm{PT}$ and $\mathrm{PZN}-x \mathrm{PT}$ single crystals in underwater hydrophones and projectors, and in ultrasonic transducers for medical applications [3-8].

As will be discussed in Section 2, the giant electromechanical response of $\mathrm{PMN}-x \mathrm{PT}$ and $\mathrm{PZN}-x \mathrm{PT}$ single crystals has generally been attributed to the monoclinic phases recently discovered at their morphotropic phase boundaries (MPBs), the nearly vertical boundary regions separating rhombohedral and tetragonal phases at low and high $\mathrm{PbTiO}_{3}$ contents, respectively. Here, the mirror planes of these monoclinic phases are said to act as "structural bridges $[9,10]$ " between the $\langle 111\rangle_{\mathrm{C}}$ and $\langle 001\rangle_{\mathrm{C}}$ limiting directions of the rhombohedral and tetragonal phases, respectively, allowing rotation [11] of the polar vector when an electric field is applied to a rhombohedral crystal along a non-polar $\langle 001\rangle_{\mathrm{C}}$ direction. The generally accepted paradigm, which will be explicitly laid out in Section 2, can be summarized as follows:

Polarization rotation, in the presence of one or more monoclinic phases, is responsible for the "giant" piezoelectric properties of PMN-xPT and PZN-xPT single crystals with morphotropic phase boundary 
compositions, when oriented and poled along a nonpolar $<001>_{C}$ direction.

However, as will be discussed below, there remains some debate about this "polarization rotation" model. One notable critic [12] has suggested that a monoclinic phase is "neither sufficient nor necessary" to understand the large piezoelectric response of PMN $-x \mathrm{PT}$ and $\mathrm{PZN}-x \mathrm{PT}$; it might be better to think of a monoclinic "distortion" rather than a true phase. Many authors have postulated that the monoclinic phases observed at zero-field in poled and unpoled crystals are metastable, perhaps the result of residual stresses due to the coexistence of rhombohedral and tetragonal domains or simply the domain structure itself [13-17]. Furthermore, it has been pointed out that strong crystal anisotropy of truly rhombohedral crystals, due to the near degeneracy of two or more phases near the MPB [18], is enough to explain the large piezoelectric response of PMN$x \mathrm{PT}$ and PZN $-x \mathrm{PT}$ along non-polar directions [19-22]. Elsewhere, authors have begun to dispute whether the monoclinic "phases" evidenced by high resolution diffraction and optical microscopy are truly monoclinic on a microscopic scale; according to the recent "adaptive phase [23]" model proposed by Viehland and coworkers, the monoclinic phases observed in PMN $-x \mathrm{PT}$ and PZN$x \mathrm{PT}$ at the MPB might be composed of very fine scale twins of truly rhombohedral or tetragonal material having only a volume-averaged monoclinic symmetry [24-26]. In contrast, there is also evidence from diffuse scattering measurements that the rhombohedral and tetragonal phases observed in PZT, and the rhombohedral phase in PMN$x \mathrm{PT}$, have local monoclinic symmetry [27-31]; the monoclinic phases observed at the MPB in PZT result from the condensation of the local monoclinic, disorder present in both rhombohedral and tetragonal phases $[29,30]$.

Thus, we can identify three main paradigms for the elevated piezoelectric response of PMN $-x \mathrm{PT}$ and $\mathrm{PZN}-x \mathrm{PT}$ single crystals at the morphotropic phase boundary and the nature of the monoclinic "phases" observed there. They are: (1) the polarization rotation model; (2) the monoclinic phase as a field-distorted higher symmetry phase; and (3) the adaptive phase model. The polarization rotation model will be laid out from a historical perspective in Section 2. The other two models will be described in Section 3 where evidence for local monoclinic symmetry in PMN-xPT and $\mathrm{PZN}-x \mathrm{PT}$ will also be discussed.

\subsection{Complex relaxor-ferroelectric solid solutions}

Unfortunately, concluding the debate will be a difficult task due to the sheer complexity of PMN $-x \mathrm{PT}$ and PZN $-x \mathrm{PT}$. As the solid solutions of a prototypic relaxor [32] $\mathrm{Pb}$ $\left(\begin{array}{lll}\mathrm{Mg}_{1 / 3} & \mathrm{Nb}_{2 / 3}\end{array}\right) \mathrm{O}_{3}[\mathrm{PMN}]$ or $\mathrm{Pb}\left(\mathrm{Zn}_{1 / 3} \mathrm{Nb}_{2 / 3}\right) \mathrm{O}_{3}[\mathrm{PZN}]$ with ferroelectric $\mathrm{PbTiO}_{3}[\mathrm{PT}]$, they show a gradual progression from relaxor to ferroelectric behavior with increasing PT content across the phase diagram [22, 33-38]. At high PT contents, the crystals show a sharp phase transition with a dielectric peak like that observed in lead titanate itself and other simpler perovskites [39]. At lower PT contents the phase transition is "diffuse [33, 40]," characterized by a frequency dependent peak in permittivity which does not correspond to a structural phase transition [41]. Just below this, a second dielectric anomaly (a "kink" or "shoulder") where all frequency dispersion disappears is evidence of a spontaneous transition to a long-range ordered structure [36], a "relaxor to ferroelectric phase transition [42]," below which the crystal is polar. Upon heating, the same kink corresponds to the "depoling temperature [34]" $T_{d}$, above which long range, ferroelectric order is lost.

As in pure PMN [43], at even lower PT contents in $\mathrm{PMN}-x \mathrm{PT}[44,45]$ and $\mathrm{PZN}-x \mathrm{PT}[46,47]$, there is debatably $[41,48-50]$ no spontaneous transition to a long range ordered, rhombohedral phase and crystals need to be poled to render them ferroelectric. However, poling using different poling protocols (e.g. field cooling), at different temperatures and differing electric field levels can often alter the resultant zero-field phase [22, 51, 52]. Furthermore, poling by field cooling can induce different phases in differently-oriented crystals; for example, $[001]_{\mathrm{C}}$-poled PMN-35PT has been shown to be monoclinic whereas $[101]_{\mathrm{C}}$-poled PMN-35PT is orthorhombic [53, 54]. As a result, $\mathrm{Cao}$ and coworkers discuss a "fragile phase stability" in PMN $-x \mathrm{PT}$ depending not only on small changes in field, but on the direction of applied field as well [54].

All measurements are also hindered by the fact that compositional heterogeneity is very difficult to avoid in solution grown crystals [55-57]. Firstly, this means that nominal compositions quoted are often misleading making systematic comparison of published results difficult. Secondly, due to the near degeneracy of the ferroelectric phases at the MPB, coexistence of two or more phases is often observed, especially in the unpoled state [15, 58-61].

Moreover, the microstructures of PMN $-x \mathrm{PT}$ and PZN$x \mathrm{PT}$ are exceedingly complex. On a microscopic scale (above one micron), large ferroelastic domains can be observed, optically, in poled and unpoled crystals with MPB compositions [62-66]. At lower and lower PT contents, these domains become smaller and less well defined forming a "tweed [67]-like" structure common to ferroelastic martensites at intermediate compositions before disappearing completely $[64,68-70]$; in PZN, for example, no domain wall contrast can be seen in the zero field cooled (unpoled) state [71]. Such a gradual change in microstructure is consistent with the transition from relaxor to ferroelectric behavior across the phase diagram. 
On a finer scale, atomic and piezo-force microscope (AFM) images show a "fingerprint" structure of $180^{\circ}$ domain walls embedded in these larger macroscopic domains [72-75], such that we can talk about a "hierarchical [69]" structure, which fragment into sub-micron sized polar "nanodomains $[69,76,77]$ " at lower PT contents. Notably, however, most microstructural studies have been done without due deference to the surface state of the crystal. Importantly, fine tweed-like structures have been shown to coarsen into proper ferroelectric domains if properly annealed to remove surface damage from the polishing process [22, 64]; tweed-like microstructures can also be removed by poling [22]. The orientation of the fingerprint patterns observed has very recently been shown to follow the direction of polishing [78].

Not least, the presence of polar nanoregions (PNRs), regions of locally correlated, short-range ferroelectric order, at temperatures high in the paraelectric phase, is an important feature of relaxor-ferroelectric PMN- $x \mathrm{PT}$ and PZN- $x$ PT $[45,79,80]$. PNRs nucleate at temperatures high above the spontaneous onset of long-range ordered ferroelectricity and their nucleation, growth and subsequent coalescence into long-range ordered rhombohedral, orthorhombic, monoclinic or tetragonal phases remains the subject of much investigation [45, 47, 48, 80-84]. Notably, they are far too small to be imaged, especially at high temperatures, and information about their size and local symmetry is most often derived from diffuse neutron or X-ray scattering experiments. Therefore, their part in the spontaneous transition to a long-range ordered phase and the hierarchical structure of embedded polar "nanodomains" observed by AFM [69, 76, 77] remains to be observed and, indeed, fully understood. Unfortunately, such diffraction studies often draw conflicting results. For example, although PMN remains short range ordered down to very low temperature $[43,48]$, there remains much debate as to whether a fully long range ordered rhombohedral phase develops in PZN upon cooling, or not [41, 46, 49, 50]. Recently, Xu and coworkers have called into question the model of PNRs as precursors, or "embryos," for the low temperature, longrange ordered, ferroelectric phase; instead, their diffuse scattering study of PZN suggests that PNRs persist, embedded in the matrix of the low-temperature, rhombohedral phase, with local polarizations perpendicular to the global $[111]_{\mathrm{C}}$ polar axis [80].

In any case, the presence of polar nanoregions is in keeping with the important disordered nature of relaxorferroelectrics in general. Thus, it is perhaps not too surprising that the presence of locally disordered, monoclinic symmetry has been evidenced in the rhombohedral phase of $\mathrm{PMN}-x \mathrm{PT}[10,31]$. The possibility that the "average" symmetries of PZT, PMN $-x \mathrm{PT}$ and PZN $-x \mathrm{PT}$ are different to their "local" symmetry at a unit cell level will be discussed in Section 3.

\subsection{Picturing the elephant}

Importantly, no single review could possibly do justice to the vast body of work already published in the field. Literally hundreds of articles have appeared on the synthesis, properties and structure of PMN $-x \mathrm{PT}$ and PZN$x$ PT since their "rediscovery [1]" in 1997 and it is now becoming more and more difficult to keep abreast of all the collected knowledge in the field. One of the major problems is that the articles have come from many differing perspectives: crystallographers have presented important high resolution X-ray and neutron diffraction data on the crystalline structure of $\mathrm{PMN}-x \mathrm{PT}$ and $\mathrm{PZN}-x \mathrm{PT}$ at the (apparent) Angstrom scale; microscopists have published transmission electron microscopy (TEM), atomic force microscopy (AFM) and polarized light microscopy (PLM) images of their microstructure at the nanometer and micron scale; and specialists in ferroelectric and piezoelectrics have reported bulk, macroscopic measurements of their dielectric, pyroelectric, ferroelectric and piezoelectric properties. Furthermore, these studies have been made: for a variety of crystal compositions in both poled and virgin states; for various crystallographic orientations; at zero field and under electrical bias; under various mechanical stresses for varying loading geometries; at various temperatures from high in the paraelectric phase down to cryogenic temperatures; and, finally, on crystals with various thermal, electrical and mechanical histories. Few authors have made systematic studies over a range of compositions; fewer still have combined bulk characterization, microstructural and high-resolution diffraction studies together. In many ways, therefore, researchers in the field are like the protagonists in the parable of the four blind men and the elephant:

Four blind men encounter an elephant. One grabs the leg and concludes it is a tree trunk. One holds the tail thinking it is a whip. Another touches the elephant's trunk and decides it's a hose and the fourth man pats the side concluding it's a wall.

All are right, of course, though none completely. We have yet to picture the whole beast.

\section{The polarization rotation model: a historical perspective}

We start with a brief historical review of the complex relaxor-ferroelectric single crystals, $\mathrm{PMN}-x \mathrm{PT}$ and $\mathrm{PZN}-$ $x \mathrm{PT}$, and thus the development of the polarization model described briefly in Section 1. Some of this section has 
been covered in other review articles, most notable in those by Park and Hackenberger [2] and Noheda [9]. Furthermore, the reader is pointed towards a number of reviews on the physics of relaxor-ferroelectrics in general [32, 85-90], especially PMN and PZN, and another excellent review article recently published by Noheda and Cox [10]. For completeness we start near the beginning, with lead zirconate titanate.

One of the biggest developments in the field of piezoelectrics came in the early 1950s with the discovery of ferroelectricity in "PZT" $\left[\mathrm{Pb}\left(\mathrm{Ti}_{x} \mathrm{Zr}_{1-x}\right) \mathrm{O}_{3}\right]$, the solid solution formed between lead zirconate $\left(\mathrm{PbZrO}_{3}\right)$ and lead titanate $\left(\mathrm{PbTiO}_{3}\right)$; lead zirconate titanate, doped with various additives, has remained the dominant piezoelectric ceramic in transducer and actuator technologies ever since [9]. The phase diagram for PZT has been refined over many years and the generally accepted version is that found in Jaffe, Cook and Jaffe (1971) [39]; it is shown in Fig. 1.

The nearly vertical, or temperature-independent, phase boundary between the low lead-titanate content $3 \mathrm{~m}$ rhombohedral phase $(\mathrm{R})$ and the high lead-titanate content $4 \mathrm{~mm}$ tetragonal phase $(\mathrm{T})$ is termed the morphotropic phase boundary (MPB) [39], meaning literally "the boundary between two forms [91]." It is the most technologically significant part of the phase diagram with the strongest dielectric, piezoelectric and coupling coefficients being found at nearby compositions [92]: piezoelectric coefficients $\left(d_{33}\right)$ ranging from around 200 to $750 \mathrm{pm} / \mathrm{V}$ can be achieved with different dopants [93] compared to around $2 \mathrm{pm} / \mathrm{V}$ in quartz.

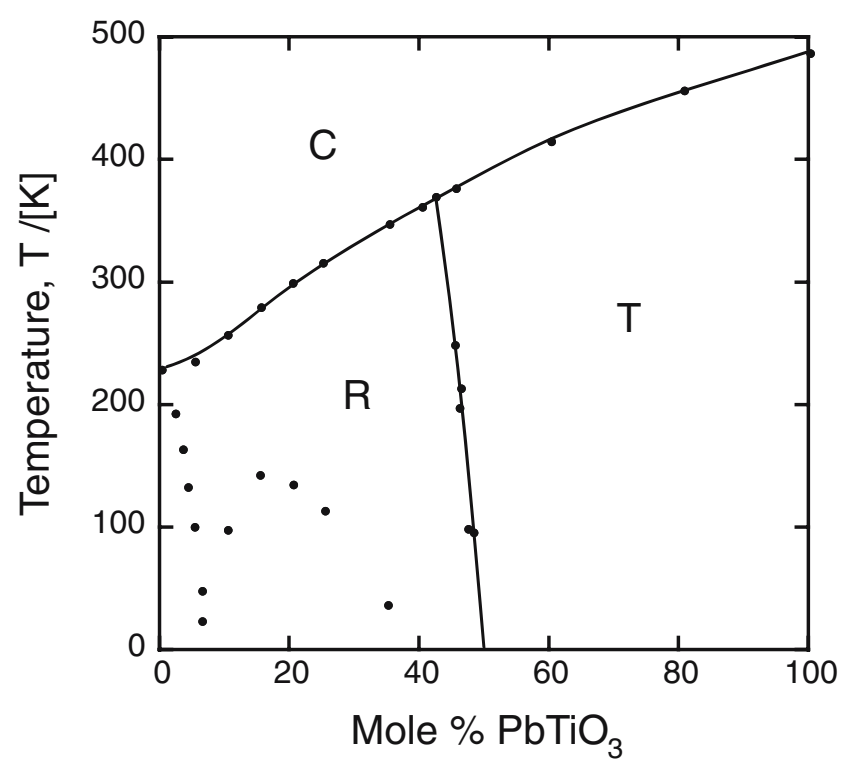

Fig. 1 The low temperature phase diagram for lead zirconate titanate (PZT) showing the cubic paraelectric phase (C) and tetragonal (T) and rhombohedral (R) ferroelectric phases. Data taken from Jaffe et al. (1971) [39]
However, despite significant scientific interest, the exact nature of the MPB and the reason for its enhanced properties remained for a long time poorly understood. Various models have been proposed including those of a finite region of two-phase coexistence, where perhaps one phase is metastable, and a true boundary where the observed coexistence can be explained by unwanted compositional fluctuations $[9,94]$. Indeed, a recent article has reiterated the thermodynamic necessity of a two-phase region at the MPB wherever diffusion is fast enough to occur during processing [94]. It is not surprising, therefore, that the width of the two-phase region in ceramic PZT has been found to depend on the processing conditions used [29].

Importantly, it was believed for a long time that such a two-phase coexistence gives rise to stronger piezoelectric response in the poled ceramic due to "an increased ease of reorientation during poling [39]." That is, the coexistence of rhombohedral and tetragonal phases presents a greater number of possible domain variants and hence a better "polarizability": that is, a greater volume of material can align their polar vectors more closely to the poling direction. This has found theoretical backing in a recent article by $\mathrm{Li}$ and coworkers [95]. However, as will be discussed in Section 3, this explanation ignores the fact that the highest piezoelectric and dielectric coefficients of a perovskite single crystal are most generally found along directions away from the polar axis [18, 96].

Most importantly for the story of PMN $-x \mathrm{PT}$ and PZN$x \mathrm{PT}$, PZT has proved nearly impossible to grow in single crystal form (except possibly by certain solid state techniques) [97]. Because of this, and due to the technological prominence of PZT polycrystalline ceramics, research into single crystal piezoelectrics took a back seat for many years.

\subsection{Relaxor-ferroelectrics}

In 1958 Smolenskii and Agranovskaya [98] reported unusual dielectric behavior in complex perovskites with the general formula $\mathrm{Pb}\left(\mathrm{B}, \mathrm{B}^{\prime \prime}\right) \mathrm{O}_{3}$, where $\mathrm{B}$ ' is a low valence cation (e.g. $\mathrm{Mg}^{2+}, \mathrm{Ni}^{2+}, \mathrm{Fe}^{3+}$ ) and $\mathrm{B}$ " is a high valence cation (e.g. $\mathrm{Nb}^{5+}, \mathrm{Ta}^{5+}, \mathrm{W}^{6+}$ ). Later named relaxorferroelectrics [32], these materials exhibit broad, diffuse peaks in permittivity at what was thought to be the phase transition between high- and low-symmetry phases, quite different to the sharp peak observed in the simpler perovskite ferroelectric, barium titanate [39]. Of such materials, lead magnesium niobate "PMN" $\left[\mathrm{Pb}\left(\mathrm{Mg}_{1 / 3} \mathrm{Nb}_{2 / 3}\right) \mathrm{O}_{3}\right]$ proved to be of most interest, having an anomalously high dielectric constant at room temperature just above a broad dielectric maximum at around $0{ }^{\circ} \mathrm{C}[32,99]$.

In contrast to barium titanate, PMN retains an averagely cubic $(\mathrm{m} 3 \mathrm{~m})$ symmetry down to around $5 \mathrm{~K}$ [41] and hence 
is not piezoelectric. However, it was found to exhibit a strong and usefully anhysteretic electrostrictive effect [100] mainly due to the high polarizability of the relaxor state [101]. It was suggested that this could be exploited in specialized actuator and transducer applications such as micropositioners and adaptive optic systems where its relatively small thermal expansion coefficient would also be an advantage [101].

Furthermore, it was found that the dielectric constant of PMN could be increased by doping with another perovskite, lead titanate; addition of lead titanate, which transforms from its paraelectric phase at around $490{ }^{\circ} \mathrm{C}$ [39], actually increases the temperature of maximum permittivity in PMN to above room temperature. Moreover, the electrostrictive coefficients of the resultant $(1-x) \mathrm{Pb}$ $\left(\mathrm{Mg}_{1 / 3} \mathrm{Nb}_{2 / 3}\right) \mathrm{O}_{3}-x \mathrm{PbTiO}_{3}[\mathrm{PMN}-x \mathrm{PT}]$ solid solution were found to increase with increased PT content, with the strain remaining predominantly electrostrictive to around $13 \mathrm{~mol} \%$ PT [101].

In 1982, a new method for synthesis of ceramic PMN and other complex mixed oxide ceramics was devised by Swartz and Shrout, which greatly reduced the presence of unwanted pyrochlore phase [102]. Usefully large pyroelectric coefficients were also identified in poled PMN $-x$ PT ceramics, which thus appeared a promising material for pyroelectric point detectors [103].

It was also discovered that upon further addition of lead titanate to PMN $-x$ PT more "classical" ferroelectric behavior is observed. Upon poling samples of a large enough PT content, PMN $-x \mathrm{PT}$ assumes a rhombohedral $(3 \mathrm{~m})$ ferroelectric phase, and therefore becomes piezoelectric. The entire phase diagram for $\mathrm{PMN}-x \mathrm{PT}$ was subsequently mapped out for ceramic PMN-xPT [99, 104] in 1989 revealing a morphotropic phase boundary between $3 \mathrm{~m}$ rhom- bohedral and $4 \mathrm{~mm}$ tetragonal phases very similar to that in PZT. Again, the piezoelectric coefficient and the relative permittivity were found to peak at the MPB, at around $700 \mathrm{pm} / \mathrm{V}$ and 5,000, respectively [104].

\subsection{Relaxor-ferroelectric single crystals}

The useful properties of relaxor-ferroelectric single crystals were first reported by Nomura and coworkers around the early 1970s. This time, the solid solution involved was based on another (albeit related) relaxor, lead zinc niobate "PZN" $\left[\mathrm{Pb}\left(\mathrm{Zn}_{1 / 3} \mathrm{Nb}_{2 / 3}\right) \mathrm{O}_{3}\right]$; PZN exhibits a diffuse phase transition closer to $100{ }^{\circ} \mathrm{C}$ and is rhombohedral when poled at room temperature [71]. Although polycrystalline samples are difficult to synthesize [33], single crystals of the lead zinc niobate lead titanate solid solution $\left[(1-x) \mathrm{Pb}\left(\mathrm{Zn}_{1 / 3} \mathrm{Nb}_{2 / 3}\right.\right.$ $\mathrm{O}_{3}-x \mathrm{PbTiO}_{3}$ or "PZN- $-x \mathrm{PT}$ "] could be readily grown by a flux method. The solution was found to exhibit a morphotropic phase boundary, very similar to that found in polycrystalline PZT and PMN $-x$ PT, between rhombohedral and tetragonal phases at around $9 \mathrm{wt} . \% \mathrm{PbTiO}_{3}$; it is shown in Fig. 2. Again, as in PZT and PMN $-x \mathrm{PT}$ ceramics, dielectric and piezoelectric properties were found to peak close to this phase boundary.

In the early 1980s, Nomura revisited PZN-xPT [19, 105]. Single crystals were grown and oriented along both $[111]_{\mathrm{C}}$ and $[001]_{\mathrm{C}}$ directions. Although modest piezoelectric coefficients were found in $[111]_{\mathrm{C}}$-poled crystals, "giant" $d_{33}$ coefficients $>1500 \mathrm{pm} / \mathrm{V}$ were measured in rhombohedral compositions near the MPB, cut and poled along the non-polar $[001]_{\mathrm{C}}$ direction.

Critically, such behavior might have been unexpected by users of poled ceramic piezoelectrics [39] where properties are always measured, necessarily, along the polar (poling)
Fig. 2 Original phase diagrams showing the position of the MPB in PZN-xPT (left) and PMN-xPT (right) single crystals taken from Kuwata et al. (1981) [105] and Shrout et al. (1990) [106], respectively. The transition temperatures plotted are taken from dielectric measurement on $[001]_{\mathrm{C}}$-oriented crystals
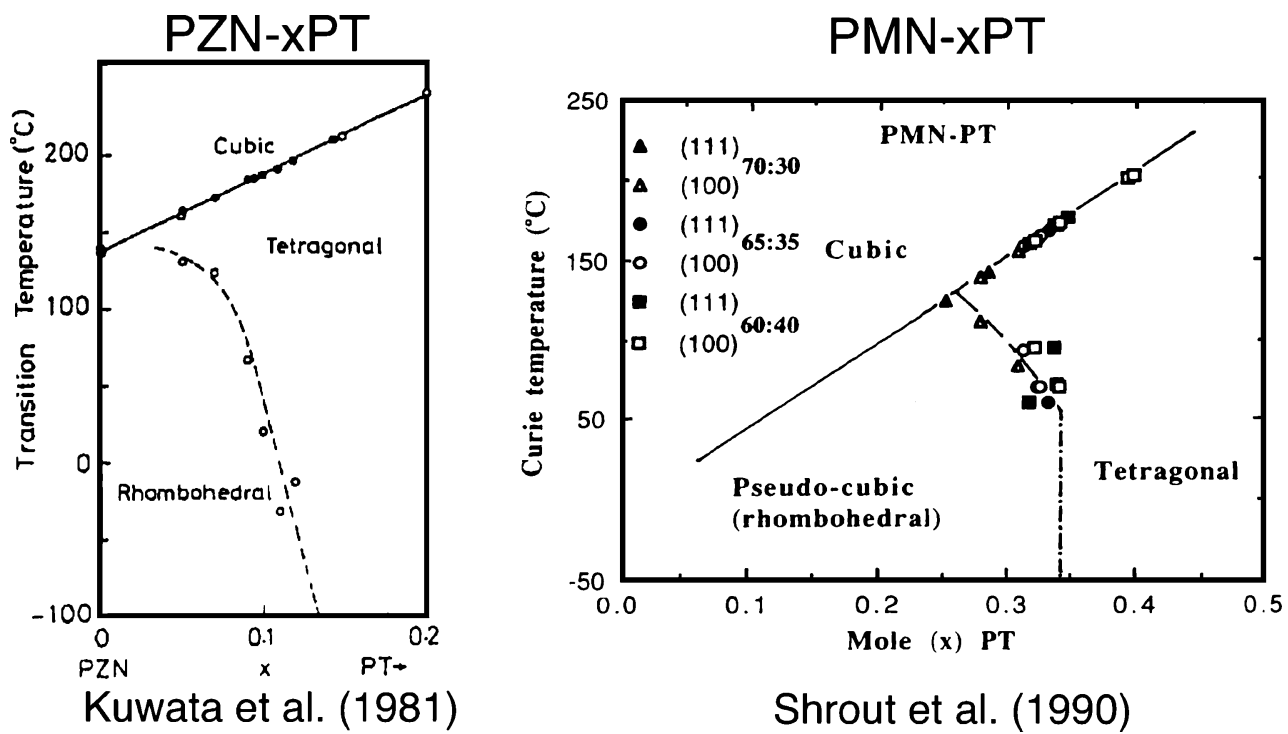

Shrout et al. (1990) 
axis and values of around $700 \mathrm{pm} / \mathrm{V}$ are achieved [104]. Notably, the authors explained the discrepancy between polar and non-polar orientations by a strong crystal anisotropy, as might be expected from basic propertysymmetry relationships in crystalline materials [96] and, especially, the large piezoelectric shear coefficient $d_{15}$ of the monodomain $[111]_{\mathrm{C}}$-oriented crystal [19]; we will return to the importance of single crystal anisotropy in Section 3.

Likewise, studies of the PMN- $x$ PT solution had hitherto only been carried out on polycrystalline samples when, in 1990, Shrout et al. published a series of measurements on flux-grown crystals with a range of compositions close to the MPB $(0.3 \leq x \leq 0.4)$ [106]. The published phase diagram, based on dielectric measurements, is also reproduced in Fig. 2. Similar to the findings of Kuwata et al., the largest piezoelectric coefficients and dielectric constants were observed in rhombohedral crystals with MPB compositions oriented along the non-polar $[001]_{\mathrm{C}}$ direction. A piezoelectric coefficient $d_{33}$ of $1,000-1,500 \mathrm{pm} / \mathrm{V}$ was reported for $[001]_{C}$-poled PMN-30PT, around five times higher than that measured along the $[111]_{\mathrm{C}}$ polar axis $(300 \mathrm{pm} / \mathrm{V})$, and twice as high as that measured in poled ceramics.

\subsection{A new era of relaxor-ferroelectric single crystals}

Later, in 1997, Park and Shrout [1] reported a series of systematic measurements of the piezoelectric properties of $\mathrm{PMN}-x \mathrm{PT}$ and $\mathrm{PZN}-x \mathrm{PT}$ single crystals cut and poled along a variety of different crystallographic directions. Not only were piezoelectric coefficients $\left(d_{33}>2,500 \mathrm{pm} / \mathrm{V}\right)$ and electromechanical coupling factors $\left(k_{33}<0.94\right)$ highest in $<001{ }_{\mathrm{C}}$-oriented rhombohedral crystals, anhysteretic strainfield responses were also observed.

One of the main drawbacks of PZT is that to achieve the highest piezoelectric coefficients, doping with additives is needed [39]; the strain-field loops in such "soft" PZT ceramics are characteristically hysteretic due to a large extrinsic contribution, typically from domain wall motion [107], resulting in poor positioning accuracy, high dielectric loss and hence significant heat generation during use [1]. Although the piezoelectric response to a unipolar electric field applied along the polar $[111]_{\mathrm{C}}$ direction was found to be weak and hysteretic, anhysteretic strains $<0.6 \%$ were observed in $[001]_{\mathrm{C}}$-poled samples of rhombohedral PZN$x \mathrm{PT}$ and $\mathrm{PMN}-x \mathrm{PT}$ [1]. Such attractive behavior is observed in rhombohedral PZN-5PT, for example, as shown in Fig. 3, indicative of minimal extrinsic, domain wall contribution to the piezoelectric response.

The absence of domain wall motion was explained by the stable domain structure formed by poling a rhombohedral crystal, containing eight possible ferroelectric domain variants [108, 109] (or orientation states) with spontaneous polarization vectors $\boldsymbol{P}_{S}$ along the $\langle 111\rangle_{\mathrm{C}}$ directions, along a non-polar $\langle 001\rangle_{C}$ direction. For example, when a sufficiently strong electric field is applied along the $[001]_{C}$ direction, four degenerate domain variants will be stabilized, with polar vectors along the $[111]_{\mathrm{C}},[1 \overline{1} 1]_{\mathrm{C}},[\overline{1} 11]_{\mathrm{C}}$ and $[\overline{11} 1]_{\mathrm{C}}$ directions. This is also shown in Fig. 3. Subsequent application of an applied field to this engineered-domain [1] structure will not favor any one domain state over the others and no domain wall motion will occur: the polar vectors in each domain variant will simply rotate continuously towards the direction of applied field (polarization rotation or "inclination [1]"). The lack of domain wall motion was later confirmed in situ by polarized light microscopy [110].

According to the model, at higher electric-fields where polarization rotation ceases, an electric-field induced phase transition to a tetragonal phase will occur where all polarizations "collapse into the $[001]_{\mathrm{C}}$ direction [1]." This too was evident in hysteretic strain-field loops at high fields, indicative of a first order, rhombohedral-to-tetragonal phase transition $[1,111]$. A subsequent in situ X-ray diffraction study by Durbin et al. (1999) [112] showed that the hysteretic strain observed in such high-field strain loops for $[001]_{\mathrm{C}}$-oriented, rhombohedral "PZN-8PT" was well reflected in the deformation of the crystal lattice and, therefore, almost entirely intrinsic in nature. This was further evidence for the inherent stability of the domain engineered structure.

\subsection{New monoclinic phases and polarization rotation}

At around the same time, something quite unexpected happened: a monoclinic phase was observed by high resolution X-ray diffraction in morphotropic phase boundary PZT (with a PT content of $48 \mathrm{~mol} \%$ ) just below room temperature [Noheda et al. (1999)] [29, 113]. High quality, homogeneous ceramic samples were used, with composi-
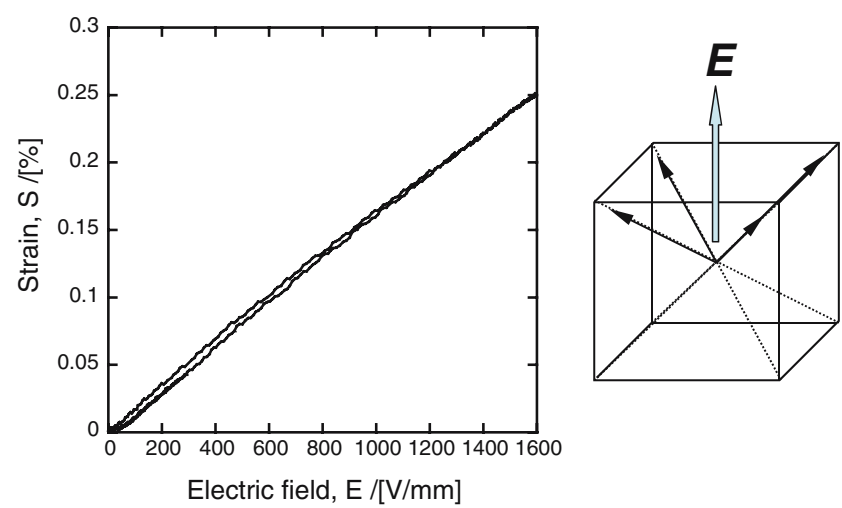

Fig. 3 Characteristic converse piezoelectric response to an unipolar electric field in a $[001]_{\mathrm{C}}$-oriented sample of PZN-5PT at $1 \mathrm{~Hz}$ and $30{ }^{\circ} \mathrm{C}$. The details of the measurement are given elsewhere [154] 
tional fluctuations less than $0.3 \mathrm{~mol} \% \mathrm{PT}$; according to the authors, the diffraction pattern observed could not be accounted for by two-phase coexistence [10,91]. After further investigation of other compositions, the monoclinic phase was subsequently found to occupy a narrow "triangle [9]" in between the rhombohedral and tetragonal phase fields of Fig. 1. At $20 \mathrm{~K}$, the monoclinic phase was found for compositions $0.46 \leq x \leq 0.51$ [Noheda et al. (2000)] [91].

The unit cell of the new monoclinic (M) phase (space group $C m$ ) is such that $a_{\mathrm{M}}$ and $b_{\mathrm{M}}$ lie along the $[\overline{110}]_{T}$ and $[1 \overline{1} 0]_{T}$ directions of the higher-temperature tetragonal (T) phase (with $a_{\mathrm{M}} \approx b_{\mathrm{M}} \approx a_{T} \sqrt{2}$ ) and $c_{\mathrm{M}}$ deviates from $[001]_{T}$ by a small angle $\left(\beta-90^{\circ}\right)$ [91], where $\beta$ is the monoclinic angle $\left(90.5^{\circ}\right.$ at $\left.20 \mathrm{~K}\right)$. Critically, in this phase (later named " $\mathrm{M}_{\mathrm{A}}$ ") [114], the only remaining symmetry element is a single $\{1 \overline{1} 0\}_{C}$ mirror plane. Therefore, instead of being fixed to lie along a single direction, the polar vector $\boldsymbol{P}_{S}$ is crystallographically free to rotate within this plane between $[111]_{\mathrm{C}}$ and $[001]_{\mathrm{C}}$ limiting directions [115]; this can be seen in Fig. 4. Further structural refinement [29] of the $\mathrm{Pb}$ cation shifts at the same temperature showed that the polar vector was rotated by about $24^{\circ}$ from the $[001]_{\mathrm{C}}$ direction towards $[111]_{\mathrm{C}}$.

$\mathrm{Du}$ et al. had already shown using phenomenological Landau-Ginzburg-Devonshire theory [116] that in PZT, as in $\mathrm{PZN}-x \mathrm{PT}$ and $\mathrm{PMN}-x \mathrm{PT}$, the highest piezoelectric properties are expected for $\langle 001\rangle_{\mathrm{C}}$-oriented rhombohedral crystals with compositions close to the morphotropic phase boundary. Again, as observed for PZN $-x \mathrm{PT}$ and $\mathrm{PMN}-x \mathrm{PT}$ single crystals, much smaller piezoelectric coefficients were predicted for crystals oriented along the $\langle 111\rangle_{\mathrm{C}}$ polar axis. Although this result could not be confirmed experimentally (due to the lack of adequately-sized PZT single crystals [97, 117]), as suggested by Park and Shrout [1], a large piezoelectric response did seem to be generated by a "polarization rotation" mechanism where the polarization vector rotates continuously towards an electric field applied

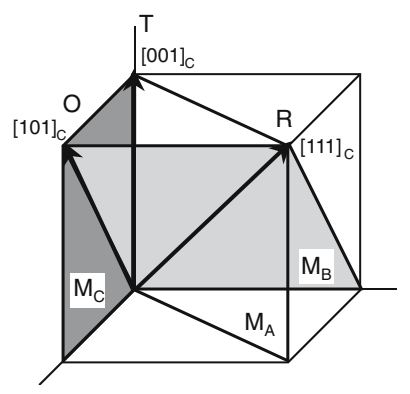

Fig. 4 Mirror planes of the monoclinic phases recently discovered at the morphotropic phase boundaries of PZT, PMN-xPT and PZN-xPT. The polar axes of the monoclinic phases are not fixed but are free to rotate within their mirror plane between the limiting directions of the rhombohedral $(\mathrm{R})$, orthorhombic $(\mathrm{O})$ and tetragonal $(\mathrm{T})$ phases. The notation is that according to Vanderbilt and Cohen (2001) [114] off-axis. Suddenly, the newly found $\mathrm{M}_{\mathrm{A}}$ monoclinic phase in PZT seemed to lend a mechanism for this rotation: i.e. the monoclinic plane acts as a "structural bridge $[9,10$, 91]", allowing or facilitating the polarization rotation, therefore leading to enhanced piezoelectric properties in PZT around the morphotropic phase boundary.

Further support for the importance of polarization rotation came from the in situ X-ray diffraction study of PZT ceramics under an applied electric field [Guo et al. (2000) [118]]. The results showed that even in averagelyoriented polycrystalline PZT, in both tetragonal and rhombohedral MPB phases, the piezoelectric distortion of the unit cell is most strongly due to rotation of the polarization vector, not its elongation. Then, in 2000, first principles calculations by $\mathrm{Fu}$ and Cohen [11] showed explicitly that in rhombohedral barium titanate (at $0 \mathrm{~K}$ ), under an electric field applied along the $[001]_{\mathrm{C}}$ direction, " $a$ large piezoelectric response can be driven by polarization rotation." Similar was later shown by the ab initio calculations of Bellaiche et al. (2001) [119] for the tetragonal and rhombohedral phases of PZT. According to these calculations, the polarization vector can also rotate continuously within the monoclinic plane as a function of composition [9], as well as upon application of a field. A breakthrough seemed to have been made: the monoclinic phase forms a convenient structural bridge across the first order, morphotropic phase boundary in PZT and also explains the elevated piezoelectric response found there [9].

Even more support for the presence of a monoclinic phase came from the theoretical work of Vanderbilt and Cohen [114] (2001). Previous phenomenological theories [120] had disallowed the presence of a monoclinic phase [9] although a fuller expansion of the Landau-GinzburgDevonshire free energy function [121] to eighth-order (as opposed to a standard sixth [120]) was found to predict not only one monoclinic phase but two, alongside the rhombohedral $(\mathrm{R})$, orthorhombic $(\mathrm{O})$ and tetragonal $(\mathrm{T})$ phases found in barium titanate [39]. The second monoclinic phase had space group $P m$, this time with the $\{010\}_{C}$ mirror plane its only remaining symmetry element. This phase, named " $\mathrm{M}_{\mathrm{C}}$," is also shown in Fig. 4. Its polar vector is constrained, by symmetry, to lie within the $\{010\}_{\mathrm{C}}$ plane between tetragonal and orthorhombic limiting directions: therefore, the orthorhombic phase is, in fact, the limiting case of the $\mathrm{M}_{\mathrm{C}}$ phase and might be termed "pseudomonoclinic [9]." A "universal phase diagram [122]" was proposed showing phase-fields for all five symmetries, R, $\mathrm{O}, \mathrm{T}, \mathrm{M}_{\mathrm{A}}$ and $\mathrm{M}_{\mathrm{C}}$, linked by various polarization rotation "paths [9]"; the simplest of such paths, R-M $\mathrm{M}_{\mathrm{A}}-\mathrm{T}$, path can be seen quite easily in Fig. 4.

Finally, one other important finding concerning the structure of PZT emerged just before the discovery of the monoclinic phase. In 1998, based on a neutron diffraction 
study, Corker and coworkers proposed that the rhombohedral phase in PZT has a locally disordered structure in which random $[001]_{\mathrm{C}}$-type $\mathrm{Pb}$ cationic shifts are superimposed upon that along the global, [111] $]_{\mathrm{C}}$ polar axis [27]; as will be discussed in Section 3.3, this implies that rhombohedral PZT has local monoclinic symmetry and only average (global) rhombohedral symmetry. Using this result, Noheda et al. proposed that the new $\mathrm{M}_{\mathrm{A}}$ monoclinic phase derives from a locally disordered rhombohedral phase in which the random, local $[001]_{\mathrm{C}} \mathrm{Pb}$ shifts condense, or freeze out, along a single direction [29].

\subsection{Monoclinic phases in PMN $-x \mathrm{PT}$ and $\mathrm{PZN}-x \mathrm{PT}$}

It was only a matter of time before monoclinic phases were also discovered at the MPBs in PMN $-x \mathrm{PT}$ and $\mathrm{PZN}-x \mathrm{PT}$ [9]. Intriguingly, it was not the $M_{A}$ phase that was found but $\mathrm{M}_{\mathrm{C}}$, in which the monoclinic $b_{M}$ axis lies instead along a $[010]_{C}$-type direction; notably, this phase is orthorhombic in its limit when $a_{\mathrm{M}}=c_{\mathrm{M}}[9,123]$. Subsequently, many high resolution (X-ray and neutron) diffraction studies [9] were carried out on single crystals, ceramics and ground powders and strong evidence was found for an intermediate $\mathrm{M}_{\mathrm{C}}$ phase in both poled [124, 125] and unpoled [123, 126, 127] samples. The monoclinic phase was also clearly identified by optical microscopy in PMN $-x$ PT [Bokov and Ye (2002)] [128] and new phase diagrams were soon published for PZN $-x$ PT [La-Orauttapong et al. (2002)] [125] and PMN$x$ PT [Noheda et al. (2002)] [126] based on the new measurements. Later X-ray diffraction work by Singh et al. [31] and Singh and Pandey [127, 129] gave evidence for the presence of an $M_{A}$ phase alongside the $M_{C}$ phase in the PMN $-x$ PT solid solution, suggesting a $\mathrm{R}-\mathrm{M}_{\mathrm{A}}-\mathrm{M}_{\mathrm{C}}-\mathrm{T}$ progression with increasing PT content. This too has recently been confirmed by careful optical microscopy [Zekria et al. (2005)] [61]. More in situ diffraction studies evidenced the same $R-M_{A}-M_{C}-T$ polarization rotation path upon application of an electric field along the $[001]_{\mathrm{C}}$ direction of MPB rhombohedral crystals [9, 130-132], the $\mathrm{M}_{\mathrm{C}}$ phase often being induced irreversibly. Finally, as in PZT, evidence was found that the local symmetry of the rhombohedral phase in PMN $-x \mathrm{PT}$ is actually monoclinic [31, 133] (Section 3.3).

Since 1997, a huge amount of work has gone into ascertaining the ferroelectric phases occurring in $\mathrm{PMN}-x \mathrm{PT}$ and PZN $-x$ PT of varying MPB compositions based mainly on high resolution X-ray or neutron diffraction $[14,53,54$, 123, 124, 134-138], optical (polarized light) microscopy [58, 61, 139-141] and bulk dielectric, pyroelectric and calorimetric measurements [142-148]. Indeed, further interest has also been rekindled in the PZT phase diagram, especially in the phases found at low temperature where octahedral tilting becomes prevalent [149].
As yet, however, no definitive phase diagram exists for PMN $-x$ PT nor PZN $-x \mathrm{PT}$, although that published recently for unpoled $\mathrm{PMN}-x \mathrm{PT}$ is perhaps the most comprehensive so far [31]. Apart from the compositional heterogeneity, as noted in Section 1, this is because unpoled crystals often lack long-range order; the "relaxor to ferroelectric transition" that occurs with increasing PT content and decreasing temperature is, by definition, diffuse such that phase boundaries are difficult to draw. In any case, such a phase diagram would not be technologically useful since unpoled crystals are not piezoelectric.

Furthermore, again as noted in Section 1, the zero-field phase present after poling and its range of thermal stability can depend upon the orientation of the applied poling field $[22,51,53,54,143,145,150]$. The importance of this result will be returned to in the Section 3. Therefore, different phase diagrams should be drawn up for the different poling directions, $[001]_{\mathrm{C}},[110]_{\mathrm{C}}$ or $[111]_{\mathrm{C}}$, as in the recent work of Cao and coworkers [54]. Because the largest piezoelectric coefficients are observed in $[001]_{\mathrm{C}^{-}}$ poled crystals it makes sense to concentrate on this orientation. Importantly, for $[001]_{\mathrm{C}}$-poled crystals, the boundary between the $\mathrm{R}$ ( or $\mathrm{M}_{\mathrm{A}}$ ) phase and the intermediate $\mathrm{M}_{\mathrm{C}}$ phase is nearly perfectly vertical in that a $\mathrm{R}-\mathrm{M}_{\mathrm{C}}$ transition is not observed upon heating in dielectric measurements, or otherwise [22, 143, 145]; instead a single phase transition to a tetragonal phase is observed. In fact, this explains why the early phase diagrams derived from dielectric measurement of $[001]_{\mathrm{C}}$-poled crystals shown in Fig. 2 fail to show an intermediate $\mathrm{M}_{\mathrm{C}}$ phase. Indeed, there is not one morphotropic phase boundary in PMN $-x \mathrm{PT}$ and $\mathrm{PZN}-x \mathrm{PT}$, but two.

\subsection{Phase transitions under external electric field and stress}

Finally, the near degeneracy of the various ferroelectric phases near the MPB means that the phase transitions between them can be induced by relatively small changes in temperature, electric field and/or stress. Since 1997, much work has also gone into investigating the various electricfield induced phase transitions that occur from the unpoled or pre-poled state when electric fields are applied to crystals of PMN $-x$ PT or PZN $-x$ PT along a non-polar $[001]_{\mathrm{C}},[101]_{\mathrm{C}}$ or $[111]_{\mathrm{C}}$ type direction. As a result, various phase transitions and "polarization rotation paths" have been evidenced by bulk strain-field measurements [151-154], and in situ diffraction [112, 130-132] and optical microscopy $[110,111,151,155-157]$. Moreover, they all share certain characteristics. They begin by the rotation of the polar vector in an $\mathrm{M}_{\mathrm{A}}\left(\mathrm{M}_{\mathrm{B}}\right)$ [54] or $\mathrm{M}_{\mathrm{C}}$ monoclinic plane (Fig. 4), which acts to reduce the angle to the applied electric field. Such polarization rotation is manifest in nearly linear, anhysteretic, macroscopic strain-field behav- 
ior like that shown in Fig. 3. Then, at a critical field, a sudden discontinuity in macroscopic strain (and polarization) and an accompanying hysteresis is observed corresponding to a first order phase transition to the field induced phase, as shown in Fig. 5. In terms of a "polarization rotation path," the abrupt change in strain corresponds to a "jump" of the polar vector either within or between monoclinic planes [154]. Physically, it corresponds to the nucleation and growth of the new phase within the matrix of the old $[151,155]$, as might be expected of a firstorder phase transition [154]. Furthermore, the induced phase can generally be predicted by simple thermodynamic arguments [154]. In some cases, more than one first order type transition can be observed in the same strain-field cycle and the transitions become less abrupt, or more diffuse, at lower PT contents or in more "relaxor" compositions where field-induced strains are more electrostrictive in nature $[22,154]$. In pre-poled samples, the initial phase is usually recovered after removal of the field. In unpoled samples, irreversible electric-field induced transitions can occur $[51,131]$, including from a short-range ordered state for samples that are not spontaneously ferroelectric [47].

Lastly, similar stress-induced phase transitions have recently been evidenced under uniaxial compressive stresses applied along non-polar directions [22, 154, 158160]; again, simple thermodynamic arguments can predict which ferroelectric (ferroelastic) phases will be energetically biased over others.

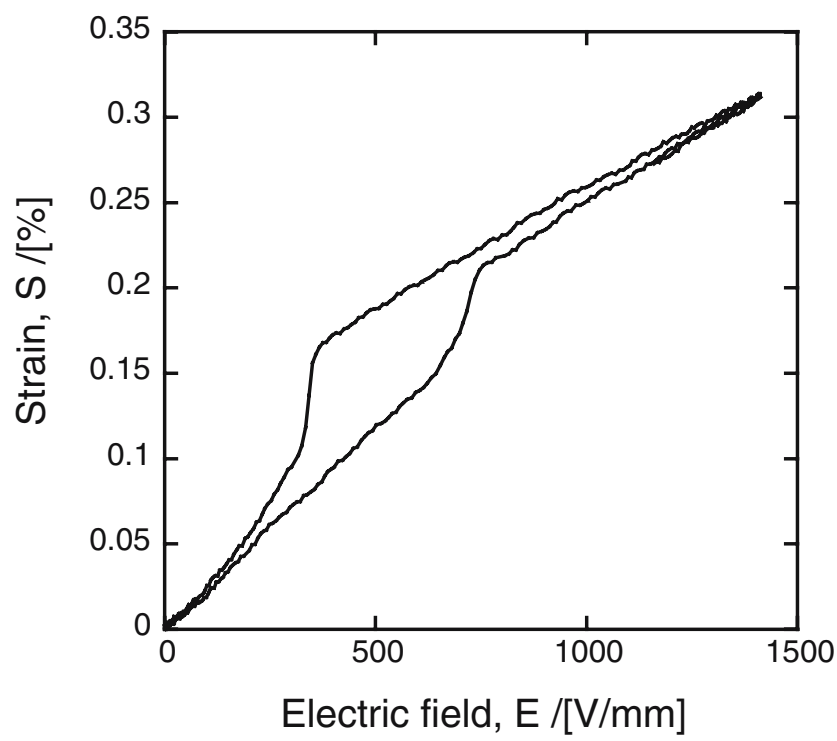

Fig. 5 Piezoelectric (strain-field) response to an unipolar electric field in a $[001]_{\mathrm{C}}$-oriented sample of PMN-31PT at $1 \mathrm{~Hz}$ and $30{ }^{\circ} \mathrm{C}$; an electric-field induced phase transition is evident. The details of the measurement are given elsewhere [154]

\section{The nature of the monoclinic phase}

To summarize, monoclinic phases having lower symmetry than the $3 \mathrm{~m}$ rhombohedral, $\mathrm{mm} 2$ orthorhombic and $4 \mathrm{~mm}$ tetragonal phases observed in the simpler perovskites $\mathrm{BaTiO}_{3}, \mathrm{KNbO}_{3}$ and $\mathrm{PbTiO}_{3}$ are readily observed in both poled and unpoled single crystals of $\mathrm{PMN}-x \mathrm{PT}$ and $\mathrm{PZN}-$ $x$ PT with MPB compositions. As implied by the polarization rotation model, these monoclinic phases act as the structural bridges necessary for rotation of the polar vector when an electric field is applied in a non-polar direction [10]. It is polarization rotation, as opposed to lengthening of the polar vector, that generates the "giant" piezoelectric response when an electric field is applied in a non-polar direction [11].

However, as will be discussed below, the true nature of these low symmetry "phases" remains in doubt. In this section, alternative paradigms to the "polarization rotation model" will be discussed.

\subsection{Monoclinic distortion in the presence of residual fields}

Importantly, the low symmetry monoclinic phases are evident in both poled and unpoled PMN-xPT and PZN$x \mathrm{PT}$ under zero external electric field and stress. Within the framework of 6th order Landau-Ginzburg-Devonshire (LGD) theory, all three monoclinic "phases" of Fig. 4 are predicted in the simpler perovskite $\mathrm{BaTiO}_{3}$ when an electric field, or stress, is applied in a non-polar direction [161]; the same will be true for the 6th order phenomenological theory of PZT. However, as noted in Section 2, an 8th order expansion is required to allow for stable, zerofield monoclinic phases [114, 162]. Recently, Bell has shown that introduction of an 8th order term to the phenomenological theory of PZT can not only stabilize a zero-field monoclinic phase at the MPB, but "enhance" polarization rotation and thus the piezoelectric coefficients predicted [30].

In spite of this, as pointed out by Kisi and coworkers [12], monoclinic phases are not necessary for polarization rotation when an electric field is applied in a non polar direction. Within the framework of 6th order LGD theory, polarization rotation will occur due to the biasing effect of an additional $-E . P$ term in the free energy expansion, where $E$ is the electric field and $P$ is the polarization; this will be true wherever an electric field is applied in a direction away from the $\langle 001\rangle_{\mathrm{C}},\langle 101\rangle_{\mathrm{C}}$ or $\langle 111\rangle_{\mathrm{C}}$ zero-field polar axis of a tetragonal $(\mathrm{T})$, orthorhombic $(\mathrm{O})$ or rhombohedral $(\mathrm{R})$ crystal, respectively [161]. When this occurs, even at an infinitesimally small field, the initial $\mathrm{T}, \mathrm{O}$ or $\mathrm{R}$ symmetry will be broken and symmetry lowering will result [12].

In fact, in truly $3 \mathrm{~m}$ rhombohedral, $\mathrm{mm} 2$ orthorhombic or $4 \mathrm{~mm}$ tetragonal crystals, polarization rotation is directly 
related, by electrostriction, to the shear coefficients ( $d_{15}$ and $d_{24}$ ) of the piezoelectric tensor [96]. Whereas the longitudinal coefficient $d_{33}$ describes extension of the polar vector, or the collinear piezoelectric effect, due to a component of electric field along the polar axis, the shear coefficients describe the shear strain due to the rotation of the polar vector towards any component of the applied field perpendicular to the polar axis: that is, polarization rotation. It is a simple exercise to show that the polar vector will rotate within a $\{010\}_{\mathrm{C}}$ or $\{110\}_{\mathrm{C}}\left(\mathrm{M}_{\mathrm{C}}\right.$ or $\left.\mathrm{M}_{\mathrm{A}}\right)$ type mirror plane of the $3 \mathrm{~m}$ rhombohedral, $\mathrm{mm} 2$ orthorhombic or $4 \mathrm{~mm}$ tetragonal phase when an electric field is applied along a non-polar $\langle 001\rangle_{\mathrm{C}},\langle 101\rangle_{\mathrm{C}}$ or $\langle 111\rangle_{\mathrm{C}}$ direction. In each case, the extent of polarization rotation will correlate directly with the shear coefficients, $d_{15}$ and $d_{24}$. Notably, as soon as polarization rotation occurs all other symmetry elements of the $\mathrm{R}, \mathrm{O}$ or $\mathrm{T}$ point group will be broken with the only remaining symmetry element a mirror plane $[12$, 161]. Therefore, the symmetry of the field distorted, lower symmetry phase will be either $\mathrm{M}_{\mathrm{A}}\left(\right.$ or $\left.\mathrm{M}_{\mathrm{B}}\right)$, or $\mathrm{M}_{\mathrm{C}}$. Such a piezoelectrically distorted $\mathrm{R}, \mathrm{O}$ or $\mathrm{T}$ phase, resulting from application of a finite field away from the polar axis, will be indistinguishable from a truly zero-field monoclinic phase.

The question therefore arises: is the field distorted structure really a monoclinic "phase"? Since electric field (or stress) is as valid a thermodynamic parameter as temperature, we might say that an electric field (or stress) induced phase transition to a monoclinic phase occurs at an infinitesimally small field. However, if such a phase transition does occur, one should also look at its order. According to group theoretical analysis [134, 162], a phase transition between $\mathrm{R}$ and $\mathrm{M}_{\mathrm{A}}$ phases can only be first order; that is, it should involve a discontinuous change in the order parameter (i.e. polarization) and should be accompanied by hysteresis when the field is removed. Therefore, an electric field induced phase transition to an $\mathrm{M}_{\mathrm{A}}$ phase cannot occur by pure polarization rotation from the zerofield $\mathrm{R}$ phase (as described by $d_{15}$ ), which by definition must be continuous. If no discontinuity is observed upon application of an electric field along the $[001]_{\mathrm{C}}$ direction, the resultant structure should be referred to as a monoclinic "distortion", rather than a "phase": the crystal's ground state remains rhombohedral.

Confusingly, in the first principles calculations of Bellaiche and coworkers for rhombohedral PZT near the MPB, application of an electric field along $[001]_{\mathrm{C}}$ does lead to a discontinuous jump in strain between $\mathrm{R}$ and $\mathrm{M}_{\mathrm{A}}$ phases indicating a true, first-order phase transition [119]. However, it was not reported in the first principles calculations of $\mathrm{Fu}$ and Cohen for low temperature, rhombohedral barium titanate for the same direction of applied field where the same $\mathrm{R}-\mathrm{M}_{\mathrm{A}}$ rotation is predicted [11]. As yet, it seems no first-order transition has been observed for the $\mathrm{R}-\mathrm{M}_{\mathrm{A}}$ rotation, either microscopically in strain-field measurements, or in situ by diffraction or optical microscopy. Experimental observation of such a transition will likely be extremely difficult due to the witheringly small lattice distortions involved as well as the fact that, in certain compositions, unpoled crystals lack long range order and poled crystals often already have zero-field monoclinic symmetry; zero-field monoclinic symmetries will be discussed below. Interestingly, however, there is now acoustic emission evidence for a first order $\mathrm{O}-\mathrm{M}_{\mathrm{C}}$ transition in PZN-9PT when an electric field is applied along the $[001]_{\mathrm{C}}$ direction [163]; according to group theory, transitions between $\mathrm{O}$ and $\mathrm{M}_{\mathrm{C}}$ phases can be first or second order [162].

As an aside, the possibility remains that if the rhombohedral phases of PMN $-x \mathrm{PT}$ and $\mathrm{PZN}-x \mathrm{PT}$ are indeed locally monoclinic the $\mathrm{R}-\mathrm{M}_{\mathrm{A}}$ transition can occur continuously without contradicting group theory [28]. That is, what would appear a $\mathrm{R}-\mathrm{M}_{\mathrm{A}}$ macroscopically would in fact correspond to a long-range condensation of a particular cation shift. This will be discussed further in Section 3.3.

From the above, we can begin to build an alternative paradigm based on truly rhombohedral, orthorhombic and tetragonal phases at the MPBs of (ferroelectric) PMN $-x$ PT and $\mathrm{PZN}-x \mathrm{PT}$. In fact, the intermediate $\mathrm{M}_{\mathrm{C}}$ phase observed in unpoled $[139,141]$ and $[001]_{\mathrm{C}}$-poled $[47,54] \mathrm{PMN}-x \mathrm{PT}$ and $\mathrm{PZN}-x \mathrm{PT}$ has orthorhombic "background symmetry [47]." That is, as mentioned in Section 2, the $\mathrm{M}_{\mathrm{C}}$ phase becomes orthorhombic in its limit [9] where $a_{M}=c_{M}$ and the polar vector lies along a $\langle 101\rangle_{C}$ direction. In general, in the $\mathrm{M}_{\mathrm{C}}$ phases observed in PMN- $x \mathrm{PT}$ and $\mathrm{PZN}-x \mathrm{PT}$, the polar vector is rotated by only a few degrees from the $\langle 101\rangle_{C}$ limiting direction. In the $\mathrm{M}_{\mathrm{C}}$ monoclinic phase discovered at very low temperature $(35 \mathrm{~K})$ in unpoled PZN-9PT by powder neutron diffraction [123], derived atomic shifts suggest the polar vector is close to $[304]_{\mathrm{C}}$. This corresponds to a rotation of around $8^{\circ}$ from the $[101]_{\mathrm{C}}$ direction. In the $\mathrm{M}_{\mathrm{C}}$ monoclinic phase evidenced in $[001]_{\mathrm{C}}$-oriented, unpoled PMN-33PT single crystals by polarized light microscopy [139], the angle of extinction is only around $3^{\circ}$ away from the position expected for an orthorhombic phase; a similar angle was found by Shuvaeva and coworkers [141].

Therefore, we might refer to such $\mathrm{M}_{\mathrm{C}}$ "phases" as "pseudo-orthorhombic [22, 51, 151]," unless of course they become "pseudo-tetragonal" in their other limit (see Fig. 4). However, when an electric field is applied to a single crystal of PZN-8PT along $[001]_{\mathrm{C}}$, the maximum angle of polarization rotation from the $[101]_{\mathrm{C}}$ limiting direction is only around $11^{\circ}$ before first order nucleation and growth of the field-induced tetragonal phase [22].

Similarly, the $\mathrm{M}_{\mathrm{A}}$ phases observed in $\mathrm{PMN}-x \mathrm{PT}$ and PZN $-x$ PT have rhombohedral background symmetry [47]. 
Typically, the polar vector is rotated only a few degrees from the $\left\langle 111{ }_{C}\right.$ limiting direction such that we might refer to such a phase as "pseudo-rhombohedral." Notably, for the $\mathrm{R}\left(\mathrm{M}_{\mathrm{A}}\right)$ - $\mathrm{T}$ transition that occurs in PZN-4.5PT upon application of a field along [001] $]_{\mathrm{C}}$, Cross and Hana [164] calculate (phenomenologically) a maximum angle of $\alpha \approx 25^{\circ}$ for rotation of the polar vector in the $\mathrm{M}_{\mathrm{A}}$ plane. Although this is not a "slight" distortion, the crystal is still "pseudorhombohedral" rather than "pseudo-tetragonal" before the phase transition.

It should perhaps be noted here that the angle of polarization rotation is distinct from the monoclinic angle $\beta$, although both can be used to quantify the "monoclinic distortion". For example, in unpoled PZN-9PT at $35 \mathrm{~K}$, the monoclinic angle is $90.25^{\circ}$, although the polar vector is rotated by $8^{\circ}$ from the $[101]_{\mathrm{C}}$ direction [123]. Because of symmetry, the angle of extinction observed in polarized light microscopy will correspond to the angle of rotation of the polar vector.

For truly zero-field rhombohedral, orthorhombic and tetragonal phases, the $\mathrm{R}-\mathrm{O}-\mathrm{T}$ sequence upon increasing $\mathrm{PT}$ content across the phase diagram neatly mirrors the sequence of phases observed in $\mathrm{BaTiO}_{3}$ and $\mathrm{KNbO}_{3}$ upon heating [39]. Within the framework of 6th order LGD theory, an orthorhombic phase is predicted in PZT close to the MPB which is only slightly higher in energy than the stable $\mathrm{R}$ and $\mathrm{T}$ phases $[165,166]$.

Importantly, it has been shown that the intrinsic piezoelectric anisotropy [96] of truly rhombohedral or orthorhombic phases can explain the majority $(>50 \%)$ of the giant piezoelectric response of $[001]_{\mathrm{C}}$-poled PMN $-x \mathrm{PT}$ and PZN $-x$ PT $[20,22,167,168]$. Huge shear coefficients $\left(d_{15}\right)$ have been measured in monodomain rhombohedral "PMN-33PT [168, 169]" (4,100 pm/V), rhombohedral PZN-7PT [170] $(7,000 \mathrm{pm} / \mathrm{V})$, and orthorhombic PZN9PT [167] $(3,200 \mathrm{pm} / \mathrm{V})$; this compares to around $560 \mathrm{pm} /$ $\mathrm{V}$ for $\mathrm{BaTiO}_{3}$ and $60 \mathrm{pm} / \mathrm{V}$ for $\mathrm{PbTiO}_{3}$, at room temperature [171, 172]. For a given electric field applied along the non-polar $[001]_{\mathrm{C}}$ direction, the resultant shear of the unit cell corresponds to large angles of polarization rotation. As noted above, the resultant symmetry under field is no longer rhombohedral or orthorhombic but strongly monoclinic.

Moreover, it has recently been pointed out that large shear coefficients are related to dielectric softening in a direction perpendicular to the polar axis, which occurs close to first order phase transitions between ferroelectric phases $[18,173]$. This includes transitions brought about by changes in temperature, as in $\mathrm{BaTiO}_{3}$ and $\mathrm{KNbO}_{3}$, stress or electric field [174], and by changes in composition, for example at a morphotropic phase boundary. In more fundamental terms, the effect is due to flattening of the free energy profile when two or more ferroelectric phases become degenerate $[107,174]$. The effect is not unique to the MPBs of PMN $-x$ PT and PZN- $-x$ PT; indeed, it has been demonstrated phenomenologically in PZT $[165,175]$. Nor is it unique to perovskites, being observed experimentally at the MPB between orthorhombic and tetragonal phases in the $\mathrm{Pb}_{1-x} \mathrm{Ba}_{x} \mathrm{Nb}_{2} \mathrm{O}_{6}$ (PBN) tungsten bronze system $[176,177]$.

However, as pointed out by Kisi and coworkers [12], the effect of the MPB and the resultant shear coefficients are uncommonly large in $\mathrm{PMN}-x \mathrm{PT}$ and $\mathrm{PZN}-x \mathrm{PT}$. Comparing the elastic constants of relaxor-ferroelectric PMN- $x$ PT [169] and PZN- $x$ PT [167] with those of $\mathrm{KNbO}_{3}$ [76], $\mathrm{BaTiO}_{3}[171]$ and $\mathrm{PbTiO}_{3}$ [178], it is clear that they are up to an order of magnitude higher than in their simpler perovskite relatives $[12,22]$. This intrinsic "softness" is likely a consequence of their background relaxor character [22]. In ferroelectric perovskites, all piezoelectric coefficients tend to increase close to the Curie point [18]; such an increase corresponds to a dielectric softening of the material in all directions. As will be discussed in Section 3.2, in relaxor-ferroelectric PMN $-x \mathrm{PT}$ and $\mathrm{PZN}-x \mathrm{PT}$, the phase transition from the high temperature, paraelectric phase is characteristically diffuse [85], or martensite-like, especially at low PT contents. Thus, the ferroelectric and paraelectric phases will remain close in energy over a large temperature range such that this dielectric softening may be felt at temperatures much below the temperature of peak permittivity.

Most importantly, it follows that monoclinic symmetries (due to polarization rotation) will also be observed in simpler perovskites when electric fields are applied in non-polar directions; however, the resultant monoclinic distortions will typically be an order of magnitude smaller, possibly beyond the resolution of most diffraction experiments.

Thus, if the zero-field, ground state phases of PMN $-x \mathrm{PT}$ and $\mathrm{PZN}-x \mathrm{PT}$ are indeed rhombohedral and orthorhombic, it is not unusual that $\mathrm{M}_{\mathrm{A}}$ and $\mathrm{M}_{\mathrm{C}}$ symmetries are observed when electric fields are applied in non-polar directions; they follow from a large piezoelectric distortion due to unusually high shear coefficients in the vicinity of the MPB. However, the presence of monoclinic phases (or distortions) in the absence of externally applied field or stress in unpoled samples is more difficult to explain as "distortions", as is the irreversible induction of monoclinic symmetry by application and removal of an applied field (poling).

Notably, two-phase coexistence is often observed in unpoled $\mathrm{PMN}-x \mathrm{PT}$ and $\mathrm{PZN}-x \mathrm{PT}$ single crystals with MPB compositions. The coexistence of rhombohedral (R) and tetragonal $(\mathrm{T})$ phases has been evidenced by polarized light microscopy (PLM), for example, in PZN-9PT and PMN-35PT [179], PZN-9PT [15], and PMN-xPT [141]. 
The coexistence of $\mathrm{M}_{\mathrm{A}}$ and $\mathrm{M}_{\mathrm{C}}$ "phases" has been observed in finely twinned by PLM in PMN-32PT [58] and a minority monoclinic phase has been reported in unpoled, tetragonal PMN-40PT [60]. Furthermore, the presence of a "trapped" tetragonal phase in unpoled, rhombohedral PZN7PT has been discussed [13]. Coexisting rhombohedral and monoclinic phases have been observed by PLM in PMN33PT [140]. Finally, the coexistence of $\mathrm{M}_{\mathrm{A}}$ and $\mathrm{M}_{\mathrm{C}}$, and $\mathrm{M}_{\mathrm{C}}$ and $\mathrm{T}$, phases has been evidenced by a Rietveld refinement of powder X-ray diffraction patterns in unpoled $\mathrm{PMN}-x \mathrm{PT}$ over certain composition ranges [127]. Such two-phase coexistence might be expected in crystals of PMN $-x \mathrm{PT}$ and $\mathrm{PZN}-x \mathrm{PT}$ due to the compositional heterogeneity discussed in Section 1 and the fact that all MPB phases are extremely close in energy. The resultant microstructure, composed of two-phase boundaries and domain walls, is generally exceedingly complex [15, 22, 141, 180].

It has been postulated by a number of authors that such two-phase coexistence leads to residual strains at the heterophase boundaries and, more importantly, that these result in symmetry lowering of the rhombohedral, orthorhombic and tetragonal phases $[13-15,58,180]$. That is, if stress-free boundaries cannot form between these phases, the resultant strains will be accommodated in the elastic distortion of the individual domains. As discussed above, for relatively small internal stresses, the resulting monoclinic distortion will be large due to the high shear coefficients and intrinsic softness of PMN $-x \mathrm{PT}$ and PZN$x$ PT. PLM studies also show that the monoclinic "distortion," determined by the rotation angle of the polar vector, can vary with location within a given domain; the variation is as much as $5^{\circ}$ in PZN-8PT [180]. This is evidence for a strongly heterogeneous strain as might be expected from a complex, two-phase, twinned structure.

A rigorous theoretical treatment of the heterophase coexistence in PMN $-x \mathrm{PT}$ and $\mathrm{PZN}-x \mathrm{PT}$ has been presented by Topolov and coworkers based on the method for calculating permissible "phase boundaries [181]" between two (or more) twinned phases original proposed by Metrat [182]; notably, the importance of monoclinic "phases" for stress relief has been pointed out. The authors have shown that a monoclinic $\mathrm{M}_{\mathrm{C}}$ phase is essential for the relief of stress in unpoled $\mathrm{PMN}-x \mathrm{PT}$ where $\mathrm{T} / \mathrm{M}_{\mathrm{C}}$ and $\mathrm{R} / \mathrm{M}_{\mathrm{C}}$ coexistence is observed [183]; similar has been shown for PZN $-x$ PT [184]. Since a monoclinic phase cannot be distinguished from a distorted, higher-symmetry one, a strained orthorhombic phase would also serve the same purpose. The importance of a monoclinic phase for elastic matching at the MPB in PZT has also been pointed out [185]. However, one problem with the Metrat approach used is that it requires experimental lattice parameters to define the deformation matrices needed. Thus, the theory alone cannot be used to predict two-phase coexistence, nor the symmetry of the phases present; it can only predict the boundaries between phases of known structure.

Another important observation is that the monoclinic "distortion" in unpoled samples, as evident in polarized light microscopy as the angle of extinction, tends to increase with temperature as the first order phase transition to a tetragonal phase (see Fig. 2) is approached [141, 180]. It also tends to increase with increasing PT content across the PMN $-x \mathrm{PT}$ and PZN- $-x \mathrm{PT}$ phase diagrams [125-127]. In the limit of the polarization rotation model, the monoclinic phase acts as a structural bridge between the rhombohedral and tetragonal phases allowing continuous rotation across the (first order) phase transition whether induced by change in temperature or composition $[9,10]$. However, such a bridge is not necessary and the increase in monoclinic distortion can also be explained by a softening of the rhombohedral or orthorhombic crystal [18] as the phase transition is approached in the presence of a constant internal stress.

In spite of this, very large, zero-field rotations of the polar vector will still be hard to explain as distortions; for example, the polar vector in unpoled $\mathrm{Pb}\left(\mathrm{Zr}_{0.52} \mathrm{Ti}_{0.48}\right) \mathrm{O}_{3}$ at $20 \mathrm{~K}$ is rotated by around $24^{\circ}$ from the $[001]_{\mathrm{C}}$ direction towards $[111]_{\mathrm{C}}[29]$, and is thus "pseudo-tetragonal" rather than "pseudo-rhombohedral." In PMN-35PT at $80 \mathrm{~K}$, the polar vector lies close to $[103]_{\mathrm{C}}$ and thus is rotated by around $27^{\circ}$ from the $[101]_{\mathrm{C}}$ direction [123]. It too is "pseudo-tetragonal," and not "pseudo-orthorhombic." Whereas a clear discontinuity in extinction angle is evidenced in PZN-8PT [180] and PMN-33PT [141] at the temperature-induced phase transition to a tetragonal phase, preceded by symmetry lowering, the rotation is nearly continuous in lower PT content PMN-28PT [141] consistent with a bridging monoclinic phase. However, the evolution of lattice parameters across the MPBs in PMN$x \mathrm{PT}$ and $\mathrm{PZN}-x \mathrm{PT}$ does not suggest truly continuous polarization rotations, nor true bridging planes [125-127]. In the original work by Noheda et al., the $\mathrm{T}-\mathrm{M}_{\mathrm{A}}$ phase transition that occurs upon cooling in $\mathrm{Pb}\left(\mathrm{Zr}_{0.52} \mathrm{Ti}_{0.48}\right) \mathrm{O}_{3}$ is clearly first order; there is a clear discontinuous jump in $\left(\beta-90^{\circ}\right)$, the relevant order parameter for the transition, at the phase transition temperature $[29,113]$.

In poled crystals, however, the picture is different. The poling process generally removes any two-phase coexistence leaving a finely twinned, single phase with a laminated, domain-engineered structure [22, 64, 66, 179, $186,187]$ based on "two-variant twinning [188]." Recently, the symmetries of the phases observed in poled PMN $-x \mathrm{PT}$ around the MPB have been shown to depend on the direction of the applied poling field. Shirane and coworkers have shown that whereas $[001]_{\mathrm{C}}$-poled (field cooled) PMN-35PT has $\mathrm{M}_{\mathrm{C}}$ symmetry, [101 $]_{\mathrm{C}}$-poled PMN-35PT is orthorhombic [53]. Furthermore, the same authors have 
shown that PMN-30PT, which is rhombohedral in the unpoled state, has $\mathrm{M}_{\mathrm{A}}$ symmetry when poled along the $[001]_{\mathrm{C}}$ direction [137]. In contrast, it assumes $\mathrm{M}_{\mathrm{B}}$ symmetry when poled along $[101]_{\mathrm{C}}$ with the polar vector rotated, within the $\mathrm{M}_{\mathrm{B}}$ mirror plane, away from the $\langle 111\rangle_{\mathrm{C}}$ limiting direction of the rhombohedral phase towards the $[101]_{\mathrm{C}}$ poling direction (see Fig. 4) [135]. All their recent results have been summarized in a recent paper; as mentioned in Section 1, the authors discuss a "fragile phase stability" of the phases around the MPB [54].

However, as has been recently pointed out [22], these results are also consistent with the model of truly rhombohedral and orthorhombic phases distorted by a residual, internal electric field or stress. Assuming the zero-field ground state of PMN-30PT is rhombohedral, upon poling along the $[001]_{\mathrm{C}}$ direction, "zero-field" $\mathrm{M}_{\mathrm{A}}$ symmetry would also result from a locked-in, internal electric field oriented along the poling direction, or a compressive stress perpendicular to it. Due to the "giant" shear coefficients of PMN $-x$ PT close to the MPB, the resultant (piezoelectric) distortion might well lie within the resolution limits of modern X-ray and neutron diffraction techniques, even for a relatively small internal bias [22].

Importantly, there is much evidence for the presence of such residual fields in poled PMN $-x$ PT and PZN $-x$ PT. First of all, the fact that $[001]_{\mathrm{C}}$-oriented crystals of various compositions can be "overpoled" to the detriment of their piezoelectric properties [52, 189] suggests that the poling process is more complicated than a simple domain switching process at the coercive field. Furthermore, there is substantial evidence that poling along the $[001]_{\mathrm{C}}$ direction increases the range of thermal stability of the high temperature tetragonal phase. For example, Sehirlioglu and coworkers have shown by dielectric measurement that in $\mathrm{PMN}-x \mathrm{PT}$ the depoling temperature $T_{d}$ is increased over that in unpoled samples by poling under a modest field at room temperature. The temperature of the lower temperature ferroelectric-ferroelectric phase transition is also lowered significantly [147]. A similar effect can also be seen in similar measurements elsewhere $[148,190]$.

In fact, an increase in thermal stability of the tetragonal phase would be expected if an external bias field were applied along the $[001]_{\mathrm{C}}$ direction. Such an electric field will favor the tetragonal phase over all other ferroelectric phases, and the cubic paraelectric phase, due to a competing energy term [161] $\Delta G=-E . P=-E P \cos \theta$; here, $\theta$ is the angle between the applied electric field $E$ and the polarization $P$ such that the phase for which $\theta$ is smallest is most strongly favored. Indeed, dielectric measurements of PMN $-x \mathrm{PT}$ under bias (that is, during field heating) show that $T_{d}$ is shifted to higher temperatures by a $[001]_{\mathrm{C}}$ electric field; the phase transition from the rhombohedral or orthorhombic phase is also moved to lower temperatures [38]. Similarly, the effect has been shown by diffraction experiments where the tetragonalcubic phase transition also becomes more second order in nature at higher bias fields [54] as observed in $\mathrm{BaTiO}_{3}$ [39].

Of course, bias fields applied along the $\langle 111\rangle_{C}$ and $<110\rangle_{\mathrm{C}}$ directions will instead favor the rhombohedral and orthorhombic phases, respectively. For example, Renault and coworkers have shown that in PZN-4.5PT, under a $[111]_{\mathrm{C}}$ bias, a direct tetragonal to rhombohedral phase transition is observed upon cooling. The same sequence of phases is observed upon zero field cooling. In contrast, under a $[101]_{\mathrm{C}}$ bias, an intermediate orthorhombic phase is seen, between the $\mathrm{T}$ and $\mathrm{R}$ phases, over a large range of temperatures (around $50{ }^{\circ} \mathrm{C}$ ) [191].

Most importantly, similar results are shown in poled samples under zero external bias. After field cooling (poling) of PZN-4.5PT along the $[101]_{\mathrm{C}}$ direction, and upon reheating under zero bias, Renault and coworkers observe the same intermediate orthorhombic phase, again stable over a large temperature range (around $20^{\circ} \mathrm{C}$ ) [191]. Interestingly, in the $[111]_{\mathrm{C}}$-poled sample, they also see evidence for an intermediate orthorhombic phase upon heating (as observed by Shen and coworkers [142]) although it is only stable over a small range of temperatures (around $5{ }^{\circ} \mathrm{C}$ ). Most importantly, the transition temperatures observed are quite different in the $[111]_{\mathrm{C}^{-}}$and $[101]_{\mathrm{C}^{-}}$-poled samples; notably, the rhombohedral phase is stable to much higher temperatures in the $[111]_{\mathrm{C}}$-poled crystal [191]. Such an effect is quite unexpected: in theory, transition temperatures should be independent of orientation.

Thus, poling along a given direction, whether by field cooling or application of a field at room temperature, seems to have the effect of leaving a "residual bias field [22]" in the material which biases the various ferroelectric phases over each other (depending on angle $\theta$ ). The effects of this can quite clearly be seen in the dielectric measurements of by $\mathrm{Lu}$ and coworkers [143, 145], and Guo and coworkers $[148,190]$. Notably, when higher poling fields are applied at room temperature, the residual biasing effect seems to be larger $[148,190]$. For example, whereas $[101]_{C^{-}}$oriented PMN-30PT is rhombohedral at room temperature when poled at $200 \mathrm{~V} / \mathrm{mm}$, it is orthorhombic after poling at $400 \mathrm{~V} / \mathrm{mm}$ [148]. This might explain the "overpoling" effects reported by Lim and coworkers [189]. The presence of residual bias fields would also explain why transitions between pseudo-rhombohedral and pseudo-orthorhombic phases are not seen in $[001]_{\mathrm{C}}$-poled crystals upon heating (Section 1), such that the MPB is nearly vertical, whereas they are in $[101]_{\mathrm{C}^{-}}$and $[001]_{\mathrm{C}^{-}}$-poled crystals [22].

Internal bias fields are not uncommon in ferroelectric materials and are found, for example, in as-grown $\mathrm{LiNbO}_{3}$ and $\mathrm{LiTaO}_{3}$ single crystals $[192,193]$ and PZT ceramics [194] due to the presence of defects. Indeed, the presence of 
a "memory effect" in $[001]_{\mathrm{C}}$-poled (field cooled) PZN-8PT, due to the alignment of defects, has been postulated by Shen and coworkers [195]. However, internal bias fields due to defects are typically apparent as a shift in bipolar, polarization hysteresis loops [194], something which is not observed in PMN $-x \mathrm{PT}$ and PZN- $x$ PT single crystals $[196,197]$. "Memory" of an externally applied field due to the presence of embedded polar nanoregions with reverse polarization, as recently evidenced by $\mathrm{Xu}$ and coworkers [198], might provide a better source for such a field. The presence of a preferred $\langle 001\rangle_{\mathrm{C}}$ poling direction in melt-grown $\mathrm{PMN}-x \mathrm{PT}$ single crystals has also been reported [199].

One important result that should be mentioned is that reported by Sehirlioglu and coworkers [199]. When [001] $\mathrm{C}^{-}$ poled PMN $-x$ PT single crystals are heated in open circuit conditions, the tetragonal-cubic phase transition evident in thermal strain measurements is shifted to higher temperatures over those seen in unpoled crystals. However, when the samples are short circuited, there is no difference in transition temperature between poled and unpoled samples. The importance of the electrical boundary conditions on measurements of phase transitions in PMN- $x \mathrm{PT}$ and PZN$x$ PT should be investigated further. Notably, dielectric measurements and in situ diffraction experiments are typically made in open circuit conditions.

Finally, such a bias effect could also come from internal stresses. For example, a compressive stress perpendicular to the poling direction would have a similar effect to an internal electric field along the poling direction. Lee and coworkers have postulated that the $\left(\mathrm{M}_{\mathrm{A}}\right)$ monoclinic distortion observed in $[001]_{\mathrm{C}}$-poled PZN-5PT results from residual strains at the domain walls of the domainengineered structure [17]. Moreover, comparing nicely with the results of Cao and coworkers [54], they find [111 $]_{\mathrm{C}^{-}}$ poled PZN-5PT to have rhombohedral symmetry [17]. Lim and coworkers have suggested that the monoclinic state found in $[001]_{\mathrm{C}}$-poled PZN-8PT is a "trapped", metastable phase [16]. The stability of the monoclinic phases observed in PZT, PMN $-x \mathrm{PT}$ and $\mathrm{PZN}-x \mathrm{PT}$ and the importance of residual stresses has recently been reviewed by Noheda and Cox [10].

In summary, the observation of monoclinic symmetries in poled and unpoled PMN $-x \mathrm{PT}$ and $\mathrm{PZN}-x \mathrm{PT}$ does not require the presence of truly zero-field monoclinic phases. They can equally be explained by the presence of residual bias fields or stresses and an intrinsic "softness" due to the proximity of the MPB, where rhombohedral, orthorhombic and tetragonal phases are nearly degenerate, and their background relaxor character. Within such a paradigm, all phase transitions between $\mathrm{R}, \mathrm{O}$ and $\mathrm{T}$ phases must be first order [162], whether induced by changes in temperature, composition, electric field or stress. There is no need for any "structural bridges" between the phases and polarization rotation under zero field within the $\mathrm{M}_{\mathrm{A}}, \mathrm{M}_{\mathrm{B}}$ and $\mathrm{M}_{\mathrm{C}}$ monoclinic planes of Fig. 4 is simply a consequence of shear deformation due to internal stresses and/or fields. The observation of monoclinic symmetries, within the resolution of optical and diffraction methods, is possible in PMN$x \mathrm{PT}$ and $\mathrm{PZN}-x \mathrm{PT}$ due to their intrinsic softness and the large resultant distortion. From this point of view, monoclinic "phases" do not explain the giant piezoelectric response at the MPB. The paradigm laid out in Section 2 is, in fact, reversed such that the monoclinic phase is a consequence of the giant piezoelectric response and not the other way around. In any case, as will be concluded in Section 4 , observations of a giant piezoelectric response and monoclinic symmetry will likely go hand in hand.

Importantly, however, the presence of residual fields remains to be confirmed. They, and the resultant monoclinic distortions they imply, should be quantified. Moreover, as pointed out by Bell, although polarization rotation does not require a monoclinic phase, it may be enhanced by the "monoclinicity" provided by an 8th order LGD term [30]; it may also be enhanced by the presence of local, monoclinic symmetry (Section 3.3). There is also the possibility that phase transitions from field-distorted $\mathrm{R}, \mathrm{O}$ and $\mathrm{T}$ phases, with monoclinic symmetry, to truly monoclinic phases do occur. At least in the case of the $\mathrm{R}-\mathrm{M}_{\mathrm{A}}$ transition, this should be first order [162]. Evidence of first order phase transitions between truly $\mathrm{R}, \mathrm{O}$ and $\mathrm{T}$ phases and their lower symmetry $\mathrm{M}_{\mathrm{A}}, \mathrm{M}_{\mathrm{B}}$ and $\mathrm{M}_{\mathrm{C}}$ counterparts would be conclusive proof for the existence of the latter. Importantly, there is now evidence for an $\mathrm{O}-\mathrm{M}_{\mathrm{C}}$ phase transition in $[001]_{\mathrm{C}}$-oriented PZN-9PT at a field of around $150 \mathrm{~V} / \mathrm{mm}$ [163], although this remains to be confirmed; notably, the transition was found to be reversible on the scale of a few days.

\subsection{Adaptive phase model}

The presence of residual bias fields was suggested [22] based on bulk (dielectric) measurements and optical microscopy; in contrast, the polarization rotation model (Section 2) is primarily based on investigation of the crystal lattice by high-resolution diffraction and first principles simulation. However, the importance of the structure at an intermediate scale, at the "mesoscale [200]" visible only by modern TEM and AFM techniques, has also begun to be recognized. Most importantly, the presence of "adaptive phases [23]" in PMN $-x \mathrm{PT}$ and $\mathrm{PZN}-x \mathrm{PT}$, similar to those commonly observed in ferroelastic martensites, has been proposed [24, 25]. Such phases, consisting of very finely twinned rhombohedral or tetragonal material, might explain the monoclinic symmetries evidenced by X-ray and neutron diffraction. 
In the "random field" theory $[45,87,89]$ of relaxorferroelectrics, the quenched electric fields created by spatial charge separation due to the presence of chemically ordered and disordered regions [89, 201] account for the lack of long-range ferroelectric order in the low temperature phases of low PT content PMN $-x$ PT and PZN- $x$ PT. In the context of this theory, random fields can be seen to frustrate the Curie-Weiss transition [39] (i.e. that seen, for example, in $\mathrm{PbTiO}_{3}$ ) and stabilize the high temperature, non-polar phase down to temperatures below the thermodynamic equilibrium temperature it would otherwise have. Noting such persistence of the parent cubic phase down to low temperatures, Schmidt [85] and others [70, 71] have pointed out the similarity between relaxor-ferroelectric and martensitic phase transitions.

Martensitic phase transitions characteristically occur far from thermal equilibrium, over a range of temperatures usually defined as the martensite start and martensite finish temperatures, $\mathrm{M}_{\mathrm{S}}$ and $\mathrm{M}_{\mathrm{F}}$, respectively [202]. Nucleation of the low temperature phase within the parent phase matrix begins at $\mathrm{M}_{\mathrm{S}}$ and becomes self-terminating at $\mathrm{M}_{\mathrm{F}}$ where some parent phase is typically left untransformed as domain boundaries between regions (domains) of the low temperature phase [85]. Growth of the martensitic phase is usually strongly anisotropic with one or more preferred growth direction [202]. Importantly, in martensites, nucleation and growth are non-equilibrium processes and the transformation can never go to completion.

It follows that the high- to low-temperature phase transitions that occur in ferroelastic martensites and relaxorferroelectrics are both "diffuse [85, 86, 203]" in that they occur over a certain temperature range, rather than at a well defined transition point. Thus, the Burns temperature [79, 204] might be suitably analogous to the martensite start temperature; the relaxor-to-ferroelectric phase transition is analogous to that which occurs at $\mathrm{M}_{\mathrm{F}}$. Moreover, the martensitic and relaxor-ferroelectric transformations share many microstructural similarities [68]. Fine (sub-micron), mesoscale, cross-hatched "tweed" microstructures have been observed in both PMN $-x \mathrm{PT}[68-70,205,206]$ and lanthanum-doped lead zirconate titanate $\left(\mathrm{Pb}_{1-\mathrm{y}} \mathrm{La}_{\mathrm{y}}\right)$ $\left(\mathrm{Zr}_{0.65} \mathrm{Ti}_{0.35}\right) \mathrm{O}_{3}$ [PLZT], another relaxor-ferroelectric [68, 70], by optical microscopy, and TEM and AFM based techniques. Similar tweed-like structures are observed in martensitic $\mathrm{Fe}-\mathrm{Pd}$ and $\mathrm{Ni}-\mathrm{Al}$ above the average transformation temperature [68]. They are also observed in the high temperature superconductor, yttrium barium copper oxide, $\mathrm{YBa}_{2} \mathrm{Cu}_{3} \mathrm{O}_{7-\delta}$ (YBCO) [200, 207, 208].

Importantly, diffuse phase transitions must involve coexistence of high temperature (parent) and low temperature (product) phases over a range of temperatures. As a result, the associated lattice mismatch between the two phases can become very important to the kinetics, thermo- dynamics and microstructural characteristics of the phase transition [23].

According to Khachaturyan and coworkers, the effect of lattice mismatch will be important to a phase transition whenever the mismatch strain energy $\mu S_{L M}{ }^{2}$ (where $S_{L M}$ is a strain characterizing the mismatch between the low temperature and parent phases and $\mu$ is a typical shear modulus) is close to or much greater than the driving force for the transformation $\Delta G_{V}$ (equal to the difference between free energies of the stress-free product and parent phases) [23]. When the mismatch energy becomes comparable to the driving force for the transition, for example in martensitic phase transitions [202], the phase transition can only proceed if complete accommodation of the lattice mismatch is provided. To achieve this, the lower temperature phase forms a complex, twinned, mesoscopic lamella structure. Importantly, the resultant volume averaged structure will be related to the parent phase by an invariant plane strain [202] (IPS); that is, to achieve energy minimization, the parent phase and the twinned product will be elastically matched to each other across a strain-free boundary (an "invariant plane" or "zero net strain plane [181]") much like domains in single phase ferroelastics [209]. When this happens, the average lamella spacing or domain size is given by [23]:

$\lambda:\left[\frac{\gamma D}{\mu S_{L M}^{2}}\right]^{1 / 2}$

where $D$ is the plate width and $\gamma$ is the domain wall energy of the low temperature phase.

During cooling, the layered, low temperature phase will eventually grow to fill the entire sample to the detriment of the surrounding untransformed parent phase [85]. Importantly, whenever the domain wall energy $\gamma$ is low, and the elastic mismatch energy $\mu S_{L M}{ }^{2}$ is large, the resultant domain size in the transformed phase $\lambda$ will be small (see equation [1]). The resultant finely-twinned, miniaturized phase is referred to as an "adaptive phase [23]". To quote Jin and coworkers [24], the adaptive phase is "a particular (miniaturized) case of conventional martensite with stressaccommodating domains, which can only be expected in situations where the domain wall energy is abnormally small". Such adaptive phases are indeed observed, experimentally, in $\mathrm{Ni}-\mathrm{Al}$ and $\mathrm{Fe}-\mathrm{Pd}$ alloys [23].

Importantly, compared to the simpler perovskites, the domain wall energies of relaxor-ferroelectric $\mathrm{PMN}-x \mathrm{PT}$ and PZN- $x$ PT are typically low [24]. This is evident in their small coercive fields, typically less than $500 \mathrm{~V} / \mathrm{mm}[196$, 210] and indicative of a high domain wall mobility, and the observation of fine, mesoscale structures themselves; for example, tweed domains of around $200 \mathrm{~nm}$ long and $20 \mathrm{~nm}$ wide have been observed in PMN-35PT [68]. It follows 
from such low domain wall energies, and the many commonalities between relaxor-ferroelectrics and martensitic ferroelastics, that the concept of adaptive phases might be relevant to PMN $-x \mathrm{PT}$ and $\mathrm{PZN}-x \mathrm{PT}$ as well.

Noticing this, Viehland and coworkers propose that the monoclinic "phases" evidenced by optical microscopy and high resolution diffraction in PMN $-x \mathrm{PT}$ and $\mathrm{PZN}-x \mathrm{PT}$ are not monoclinic at all $[24,25]$. Instead, the $\mathrm{M}_{\mathrm{A}}$ and $\mathrm{M}_{\mathrm{C}}$ states reported at the MPB are really adaptive phases composed of finely twinned rhombohedral or tetragonal phases, importantly, having only volume-averaged monoclinic symmetry. In their model, the $\mathrm{M}_{\mathrm{C}}$ "phase" observed in PMN- $x \mathrm{PT}$ and $\mathrm{PZN}-x \mathrm{PT}$ is really a tetragonal adaptive phase composed of alternating lamella of two $90^{\circ}$ tetragonal domain variants (two variant twinning [188]). In contrast, the $\mathrm{M}_{\mathrm{A}}$ "phase" observed is composed of finely twinned rhombohedral variants [24].

According to this model, the volume averaged unit cell of the finely-twinned adaptive phase, as derived from diffraction experiments with a finite beam size, will be different to the true unit cell of the lattice [26]. Importantly, it will be a function of the lattice parameters of the low temperature phase and the volume fraction $\omega$ and $(1-\omega)$ of each of the (two) component variants.

As noted above, the volume averaged unit cell of the adaptive phase must be related to the unit cell of the parent phase by an invariant plane strain (IPS). Using the Metrat method [182], the volume fraction of each variant required for an IPS can be derived as a function of the lattice parameters of the low temperature rhombohedral (R) or tetragonal (T) phase (e.g. $a_{T}$ and $c_{T}$ ) [24]. In this way, the volume average lattice parameters of the resultant adaptive phase $\left(a_{\mathrm{ad}}, b_{\mathrm{ad}}\right.$ and $\left.c_{\mathrm{ad}}\right)$ can be calculated as a function of the lattice parameters of the tetragonal or rhombohedral phases and the volume fractions of the two twins. Notably, it can also be shown that certain "invariance conditions," relating the volume average lattice parameters of the adaptive phase to those of its constituent domains, hold wherever the conditions for an IPS are met, irrespective of the volume fraction of each twin $[24,26]$. For example, for a tetragonal adaptive phase:

$$
\begin{aligned}
& a_{\mathrm{ad}}+c_{\mathrm{ad}}=a_{T}+c_{T} \\
& b_{\mathrm{ad}}=a_{T}
\end{aligned}
$$

Importantly, the polarization $P$ of the $\mathrm{M}_{\mathrm{A}}$ or $\mathrm{M}_{\mathrm{C}}$ adaptive phase, as measured by diffraction or polarized light microscopy, will be the volume average of the polar vectors of the component domains. Thus, in the case of an $\mathrm{M}_{\mathrm{C}}$ adaptive phase composed of tetragonal variants with polar vectors along $[001]_{\mathrm{C}}$ and $[100]_{\mathrm{C}}$, the volume averaged polarization $\boldsymbol{P}$ can lie anywhere between these two limiting directions, depending on the relative volume fractions $\omega$ and $(1-\omega)$. That is, the polarization vector is free to rotate within the $(010)_{C} M_{C}$ monoclinic plane (see Fig. 4) as a function of $\omega$.

Moreover, application of an electric field or a noncentrosymmetric stress, will bias one of the constituent variants over the other leading to domain switching, that is, the progressive growth of one variant at the expense of the other [23]. Thus, when an electric field is applied along $[001]_{\mathrm{C}}$, for example, the net polarization will rotate within the $(010)_{\mathrm{C}}$ plane towards that direction. Thus, the adaptive phase can not only explain the observation of monoclinic symmetries but can also explain the polarization rotation observed under field [24-26]. Furthermore, if a large enough field can eliminate one of the variants entirely a truly tetragonal phase will be observed; therefore, the adaptive phase model is also consistent with the electric field induced phase transitions first evidenced by Park and Shrout [1]. Finally, it can also explain the orthorhombic symmetry phase observed in otherwise $\mathrm{M}_{\mathrm{C}}$ compositions when poled along the $\langle 101\rangle_{\mathrm{C}}$ direction [54]; in the presence of such a poling field both tetragonal variants are degenerate and the resultant volume fraction $(\omega=1 / 2)$ will fix a volume-averaged orthorhombic symmetry [25].

Therefore, in the adaptive phase model, the huge electric-field induced strains [1] $(>1 \%)$ shown by PMN$x \mathrm{PT}$ and $\mathrm{PZN}-x \mathrm{PT}$ are not intrinsic in nature; they are instead the result of domain switching on a nanoscale. Importantly, the ease of polarization rotation is thus explained by the high domain wall mobility of the "nanotwins [26]" due to a low domain wall energy. From this point of view, monoclinic bridging phases are unnecessary and the large intrinsic piezoelectric anisotropy expected close to the MPB is redundant.

Indeed, there is strong quantitative evidence for the validity of the adaptive phase model. Firstly, the anisotropic peak broadening often observed in X-ray diffraction experiments is consistent with the presence of a highly twinned microstructure [26]. Secondly, the strain observed during the $\mathrm{M}_{\mathrm{C}^{-}}-\mathrm{T}$ electric-field induced phase transition in $[001]_{\mathrm{C}^{-}}$ oriented $\mathrm{PZN}-8 \mathrm{PT}$ is consistent with that expected from switching of tetragonal domains [26]. Thirdly, there is much evidence that the various invariance conditions (like those in equation [2]) hold in both PMN $-x \mathrm{PT}$ and PZN$x \mathrm{PT}$ across the $\mathrm{M}_{\mathrm{C}}-\mathrm{T}$ phase transition whether induced by changes in temperature, composition or electric field [26].

For an $\mathrm{M}_{\mathrm{C}}$ adaptive phase composed of tetragonal nanotwins, the second invariance condition of equation [2] can be written $b_{M}=a_{T}$, implying continuity of one of the lattice parameters across the $\mathrm{M}_{\mathrm{C}} \mathrm{T}$ phase transition. As shown by Wang [26], it is observed convincingly for a variety of PMN $-x \mathrm{PT}$ and $\mathrm{PZN}-x \mathrm{PT}$ compositions. Indeed, continuity of the smallest lattice parameter can be seen across the entire phase diagram of unpoled, room temperature PMN- $x$ PT, bridging all four $\mathrm{R}, \mathrm{M}_{\mathrm{A}}, \mathrm{M}_{\mathrm{C}}$ and $\mathrm{T}$ phases 
[26]. However, the continuity is less convincing for fieldinduced $\mathrm{M}_{\mathrm{C}}-\mathrm{T}$ transitions. At a glance, it is also less convincing across the $\mathrm{T}-\mathrm{M}_{\mathrm{C}}$ transition observed during field cooling.

The relevance of such a condition is also unclear and similar relationships should be sought in other materials. In fact, the same invariance condition is observed across the $4 \mathrm{~mm}$ tetragonal to $\mathrm{mm} 2$ orthorhombic phase transition of $\mathrm{As}_{2} \mathrm{O}_{5}$; notably, this phase transition is second order [67]. Finally, although there seem to be obvious trends in the derived values of $\omega$ for the $M_{C}$ phase under increasing electric field, there are no obvious trends for increasing composition or temperature [26]; the reason for this is not discussed.

Perhaps most importantly, as recently pointed out [22, 211], since polarization rotation is inherently coupled to local ferroelastic domain wall motion in the adaptive phase model, albeit on a very fine scale, it will be irreversible wherever domain walls are pinned by defects; that is, hysteresis and frequency dependence might be expected as a result [212]. However, no hysteresis is observed in the strain-field response of $[001]_{\mathrm{C}}$-poled PMN- $x$ PT and PZNPT. As noted in Section 2 it is characteristically anhysteretic (see, for example, Fig. 3). Moreover, the reversible contribution to the direct $d_{33}$ response of [001] $]_{\mathrm{C}}$-poled $\mathrm{PMN}-x \mathrm{PT}$ and $\mathrm{PZN}-x \mathrm{PT}$ is always much greater than any extrinsic, irreversible part [22, 213, 214]. It is unclear at this stage why progressive switching in the adaptive phase should be anhysteretic [214].

Last but not least, such fine-scale (nano-) twinning has yet to be confirmed, beyond doubt, experimentally, although features of such a hierarchical, nanoscale domain structure have recently been observed by TEM in PMN33PT by Wang and coworkers [215]. The model will certainly need reconciling with the vast amounts of evidence from TEM, SFM, and X-ray and neutron scattering and it is not clear how the adaptive phase model fits with the embedded nanodomain structures discussed in Section 1.

In any case, more work is needed to better understand the link between ferroelastic martensites and relaxorferroelectrics in general; their commonalities, discussed here and elsewhere [216, 217], do indeed seem to run deep. In fact, the similarities may extend to all ferroelectrics with some degree of compositional or structural disorder. For example, "frustrated microstructures" showing tweedlike contrast are also observed by TEM close to the morphotropic phase boundary in PZT (although again the importance of TEM specimen preparation is unclear) [149]. PZT, which also has mixed B-site occupancy, has the potential for an inhomogeneous distribution of $\mathrm{Zr}$ and $\mathrm{Ti}$ cations [218]. The importance of order and disorder in ferroics in general (that is, all ferroelectrics, ferroelastics and ferromagnetics) is a huge topic, beyond the scope of this article. However, some brief comments will be made in the next section.

\subsection{Importance of order/disorder and local} symmetry lowering

As distinct from a "displacive" phase transition, where the symmetry of the parent phase is broken by the displacement of atoms away from their high temperature positions, an "order-disorder" transition is one in which the occupancies of the lattice positions, not the positions themselves, change [200]. There is in fact significant evidence that even the simplest perovskites show some degree of order/disorder character.

Whereas lead titanate $\left(\mathrm{PbTiO}_{3}\right)$ undergoes a purely displacive phase transition [219] at its Curie point $T_{C}$ consistent with classical soft mode theory [220], two other "classical" ferroelectrics, barium titanate $\left(\mathrm{BaTiO}_{3}\right)$ and potassium niobate $\left(\mathrm{KNbO}_{3}\right)$, show evidence for a Burns temperature far above $T_{C}$, as observed in relaxorferroelectrics PMN and PZN (Section 1). Deviation from linearity of the refractive index is observed at around $300{ }^{\circ} \mathrm{C}$ in barium titanate, around $180{ }^{\circ} \mathrm{C}$ above $T_{C}$. Moreover, the phase transition from the paraelectric to the ferroelectric phase is not well described by soft mode theory [204]. Burns and Dacol state that the behavior of $\mathrm{BaTiO}_{3}$ can be better described in the "language of orderdisorder systems [204]."

Indeed, the order/disorder character of $\mathrm{BaTiO}_{3}$ and $\mathrm{KNbO}_{3}$ was first postulated by Comès and coworkers in 1970 [221] based on evidence from diffuse X-ray scattering. To explain the diffuse scattering, the authors suggested a model where only the rhombohedral ground state phase is fully ordered. In contrast, the orthorhombic, tetragonal and cubic phases are all partially disordered, formed by combinations of "chains" of unit cells with Bsite cations uniformly displaced along one of the $\langle 111\rangle_{\mathrm{C}}$ directions [222]. Elsewhere, lattice dynamics modeling has been used to explain why only the orthorhombicrhombohedral phase transition in $\mathrm{KNbO}_{3}$ fits displacive soft mode theory, while the other two transitions display both displacive and order/disorder characteristics. A "hopping" of the B-site cation in the rhombohedral phase is suggested [223]. Furthermore, a study of the microscopic and macroscopic symmetry of $\mathrm{BaTiO}_{3}$ through the phase transition has shown that some local tetragonal symmetry persists above $T_{C}$; based on this, Wada and coworkers again suggest a mixed displacive and order/disorder character [224]. Finally, the same has been suggested based on firstprinciples calculation [225].

It can be no surprise, therefore, that in the more complex relaxor-ferroelectric solid solutions, $\mathrm{PMN}-x \mathrm{PT}$ and $\mathrm{PZN}-$ 
$x$ PT, structural disorder is also evident in the presence of a polar nanoregions, and complex, frustrated microstructures. Importantly, in the solid solutions PMN- $x$ PT and PZN$x \mathrm{PT}$, and in PZT, compositional disorder will arise due to mixed occupancy of the B-site. The links between compositional and structural disorder in complex perovskite solid solutions remain the subject of much investigation [28, 88, 89, 226-230].

Notably, there is now significant evidence that in PZT, and in relaxor-ferroelectrics like $\mathrm{PMN}-x \mathrm{PT}$ and $\mathrm{PZN}-x \mathrm{PT}$, the average symmetry observed by X-ray and neutron diffraction is different to that on the unit cell level: a full review of local versus average symmetry lowering can be found in the recent article by Noheda and Cox [10].

Although only average lattice distortions can be determined by X-ray and neutron diffraction data, information about static or dynamic disorder, or local short-range order, can also be derived from analysis of so-called anisotropic displacement parameters [27, 133]. As discussed in Section 2, structural refinement of neutron diffraction data shows that the $\mathrm{Pb}$ cationic shifts in PZT might be best described as monoclinic in both rhombohedral and tetragonal phases [27, 28].

Corker and coworkers were first to propose that, in the rhombohedral phase of $\mathrm{PZT}, \mathrm{Pb}$ cation shifts can be described by a long range ordered displacement along the $[111]_{\mathrm{C}}$ direction superimposed upon local, short range ordered displacements along either a $[001]_{\mathrm{C}},[010]_{\mathrm{C}}$ or $[100]_{\mathrm{C}}$ direction [27]. Implicitly, in regions of material where the shifts are locally ordered, the average symmetry will be lowered to $\mathrm{M}_{\mathrm{A}}$ monoclinic [29]. Globally, however, the shifts are uncorrelated and the average symmetry is rhombohedral. As pointed out by Noheda and coworkers, the same local monoclinic symmetry can also result from locally correlated $[110]_{\mathrm{C}}$-type shifts superimposed upon a long-range tetragonal $[001]_{\mathrm{C}}$ shift $[29,133]$. This has led to the suggestion that the local symmetry of PZT is $\mathrm{M}_{\mathrm{A}}$ in both $\mathrm{R}$ and $\mathrm{T}$ phases [28, 133]. Average monoclinic symmetry is thus due to the condensation, or freezing out, of one of the disordered $\mathrm{Pb}$ cationic shifts [30].

In this model, structural order, in the long range correlation of the monoclinic shifts, is highest at the MPB where volume average $\mathrm{M}_{\mathrm{A}}$ symmetry is observed [28]. Therefore, the R-M $\mathrm{M}_{\mathrm{A}}-\mathrm{T}$ transition across the MPB in PZT is essentially an order-disorder transition, rather than a displacive one, and can be continuous without contradiction of group theory (Section 3.1). Local monoclinic symmetry would allow continuous polarization rotation, between the $[001]_{\mathrm{C}}$ and $[111]_{\mathrm{C}}$ limiting directions, within the same phase. The gathering evidence to support this model, from $\mathrm{X}$-ray and neutron diffraction, and first principles calculation, has recently been reviewed by Noheda and Cox [133], and by Bell [30].
Moreover, there is now evidence for local symmetry lowering in relaxor-ferroelectrics. A recent neutron diffraction study by Singh and coworkers suggests that rhombohedral PMN-25PT has local, short-range monoclinic $\mathrm{M}_{\mathrm{B}}$ $\left(\mathrm{M}_{\mathrm{A}}\right)$ symmetry, which grows to long-range monoclinic order at low temperatures [31]. Evidence for local monoclinic symmetry in the rhombohedral phase of $(1-x) \mathrm{PbSc}_{1 / 2}$ $\mathrm{Nb}_{1 / 2} \mathrm{O}_{3}-x \mathrm{PbTiO}_{3} \quad[\mathrm{PSN}-x \mathrm{PT}]$ has been presented by Haumont and coworkers [228].

The picture is complex and further discussion is far beyond the scope of this article. In PMN-xPT, for example, $\mathrm{X}$-ray photoelectron spectroscopy suggests that structural disorder increases towards the MPB [226]. Elsewhere, the importance of chemical disorder for the stabilization of monoclinic phases has also been discussed [218, 228, 231]. The links between local and average structure, structural disorder, compositional disorder, relaxor-ferroelectric behavior and the presence and structure of polar nanoregions remain to be fully understood.

Most importantly, a paradigm based on structural disorder and locally monoclinic phases remains to be reconciled with the three paradigms discussed above. However, as pointed out by Bell [30], the lateral cationic shifts responsible for local monoclinic symmetry might be expected from dielectric softening perpendicular to the polar axis close to the morphotropic phase boundary, as predicted by phenomenological theory [18]. Finally, the importance of B-site alloying to the large $d_{15}$ shear coefficient of PZT has been pointed out based on first principles calculations [231]. The large shear coefficients observed in rhombohedral PZN-xPT and PMN $-x \mathrm{PT}$ (see Section 3.3) may indeed be a direct result of locally disordered, monoclinic symmetry.

\subsection{Possible extrinsic contributions}

Until this point, the presence of a domain engineered structure $[232]$ in $[001]_{\mathrm{C}^{-}},[101]_{\mathrm{C}^{-}}$or $[111]_{\mathrm{C}^{-}}$poled PMN$x$ PT and PZN $-x$ PT has been ignored. Since no domain wall motion occurs upon application of an electric field along the poling direction (Fig. 3), the domain engineered structure has simply implied that the monodomain structure and properties of $\mathrm{PMN}-x \mathrm{PT}$ and $\mathrm{PZN}-x \mathrm{PT}$, when an electric field is applied along a non-polar direction, can be investigated using polydomain crystals. However, as will be discussed below, domain walls might well provide an extrinsic contribution to their piezoelectric response.

As noted above, intrinsic crystal anisotropy can only account for between $50 \%$ to $80 \%$ of the measured piezoelectric response of $[001]_{\mathrm{C}}$-poled $\mathrm{PMN}-33 \mathrm{PT}$ and PZN-9PT [20, 21, 167, 168]. There is evidence, therefore, of a significant extrinsic contribution ( $>20 \%)$ to the enhanced piezoelectric properties of domain-engineered 
$\mathrm{PMN}-x \mathrm{PT}$ and PZN $-x \mathrm{PT}$. However, there is no evidence for domain wall motion in strain-field measurements when an electric field is applied along the $[001]_{\mathrm{C}}$ poling direction of pseudo-rhombohedral and pseudo-orthorhombic crystals (see Fig. 3). Due to the inherent stability of the domain engineered structure, ferroelastic domain switching does not occur [1]. However, there remains the possibility that the contribution may arise purely from the presence of domain walls.

Recent work by Wada and coworkers has shown experimentally that an increased domain density leads to increased piezoelectric properties in domain engineered $4 \mathrm{~mm}$ barium titanate and $\mathrm{mm} 2$ potassium niobate [233237]. For example, in $[111]_{\mathrm{C}}$-oriented $\mathrm{BaTiO}_{3}$, the measured $d_{33}$ response of a monodomain crystal was found to be $224 \mathrm{pm} / \mathrm{V}$ [237], very close to the theoretical value calculated assuming a purely intrinsic contribution $(222 \mathrm{pm} / \mathrm{V})$. In a multidomain crystal, however, the response was found to be dependent on the average domain size, controllable via the poling technique used. Decreasing the domain size from $>30$ to $14 \mu \mathrm{m}$ led to an increase in $d_{33}$ from 241 to $289 \mathrm{pm} / \mathrm{V}$, albeit without change in permittivity. Such a $30 \%$ increase in piezoelectric response with increasing domain wall density does indeed suggest that there is a significant extrinsic response from the presence of a domain structure, especially when this structure becomes fine. As noted above, fine domain structures are often found in domain engineered $\mathrm{PMN}-x \mathrm{PT}$ and $\mathrm{PZN}-x \mathrm{PT}$, especially for low PT-contents.

The reason for this observed domain density dependence remains unclear. However, recent continuum modeling suggests that polarization vectors become more rotated from their zero-field direction in crystals with fine domain structures [238]. Moreover, first-order, electric-field induced phase transitions have been shown to nucleate at domain walls [238, 239]. Local bias fields applied antiparallel to the polarization can lead to large enhancements of the piezoelectric response in non-polar directions [240].

What is more, this extrinsic contribution might explain the fact that "giant" piezoelectric coefficients are observed even in low PT-content crystals such as PZN-4.5PT, far away from the MPB, where lower piezoelectric shear coefficients are expected [18]. A recent Brillouin scattering study, albeit in stark contrast to measurements elsewhere [241, 242], suggests a weak piezoelectric anisotropy in monodomain PZN-4.5PT $\left(d_{15} / d_{33}=328 / 202 \approx 1.6\right)$ [241]. The large piezoelectric response of $[001]_{\mathrm{C}}$-poled PZN-4.5PT $\left(d_{33}>2,000 \mathrm{pm} / \mathrm{V}\right)$ [243], might thus arise from an even larger extrinsic contribution $(>90 \%)$. Accordingly, domain sizes are shown to decrease at lower PT contents as a result of a lower domain wall energy. As discussed above, the domain engineered structure might also be responsible for the presence of residual bias fields [22].
Finally, it has been postulated that strain fields arising between the long-range ordered phase and the embedded polar nano-regions in $\mathrm{PMN}-x \mathrm{PT}$ and $\mathrm{PZN}-x \mathrm{PT}$ may also help explain their large electromechanical response [80].

\section{Concluding remarks}

Understanding the structure and properties of the relaxorferroelectric solid solutions PMN $-x$ PT and PZN $-x$ PT will remain an extremely difficult task. As noted in Section 1, they are exceedingly complex in their chemistry, and in their structure across a range of length scales. However, three main paradigms for their "giant" piezoelectric properties of PMN $-x \mathrm{PT}$ and $\mathrm{PZN}-x \mathrm{PT}$ can be identified.

In the first, laid out in Section 2, the monoclinic phases present at the morphotropic phase boundary provide "structural bridges [9]" across the first order transition between rhombohedral and tetragonal phases. Moreover, they are responsible for the giant piezoelectric response when electric fields are applied in non-polar directions in that they allow, or at least facilitate [30], the polarization rotation [11] mechanism.

In the second paradigm, described in Section 3.1, a strong polarization rotation effect is explained by the large piezoelectric shear coefficients of zero-field rhombohedral and orthorhombic phases due to the destabilizing effect of the MPB $[18,174]$ and the intrinsic softness of the relaxor state. It is noted that monoclinic phases are not necessary for the rotation of the polar vector when fields are applied in non-polar directions [12]. Zero field monoclinic symmetries can instead be explained by residually distorted rhombohedral and orthorhombic phases in the presence of internal stresses and/or residual bias fields [22].

In the third paradigm (Section 3.2), postulated by Viehland and coworkers, the monoclinic "phases" of $\mathrm{PMN}-x \mathrm{PT}$ and $\mathrm{PZN}-x \mathrm{PT}$ are, in fact, composed of very finely twinned rhombohedral and tetragonal domains [24, 25]. In this adaptive phase model, based on that for ferroelastic martensites [23], the large electric-field induced strains are extrinsic in nature and result from the progressive switching of the component "nanotwins". The ease of polarization rotation is thus explained by the high domain wall mobility of relaxor-ferroelectric PMN $-x \mathrm{PT}$ and PZN$x$ PT. Importantly, the adaptive phase model follows naturally from the many similarities between relaxor ferroelectrics and martensites in general.

Lastly, the importance of structural and chemical disorder to the structure and properties of $\mathrm{PMN}-x \mathrm{PT}$ and $\mathrm{PZN}-x \mathrm{PT}$ has been discussed. There is now significant evidence that local symmetry, on a unit cell scale, may be different to the average symmetry evidenced by diffraction experiments (Section 3.3) [10]. Local monoclinic symmetry 
in both rhombohedral and tetragonal phases of PZT would mean that the polar vector can rotate between $[111]_{\mathrm{C}}$ and $[001]_{\mathrm{C}}$ limiting directions, across the MPB, without a change of phase. Finally, evidence for an extrinsic contribution to the piezoelectric response of PMN $-x \mathrm{PT}$ and PZN $-x$ PT from the presence of domain walls has been reviewed (Section 3.4).

Importantly, all the paradigms described remain to be reconciled both with each other and with the extremely complex, disordered, relaxor nature of $\mathrm{PMN}-x \mathrm{PT}$ and PZN $-x$ PT. Which paradigm is most "correct" remains to be seen. Of course, it is possible that the different paradigms might be active in certain compositions but not in others. Above all, there is surely some truth in all of them, very much in keeping with the parable of the four blind men and the elephant. Free-energy flattening, leading to enhanced dielectric and piezoelectric anisotropy and large shear coefficients; diffuse, martensitic-like behavior, leading to mesoscale "tweed-like" structures and nanoscale twinning; and, chemical and structural disorder leading to the presence of polar nano-regions and local monoclinic symmetry may all be intrinsically related. As recently postulated by Kutnjak and coworkers, a large electromechanical response can be expected whenever a system is close to criticality [244]; all the above effects result from a proximity to some kind of instability. Which phenomena is most fundamental is likely to prove a matter of taste.

If there is one thing that should be conveyed by this article, it is huge amount of literature that has been published about PMN $-x \mathrm{PT}$ and $\mathrm{PZN}-x \mathrm{PT}$. Articles have been written from many different perspectives, based on Xray and neutron diffraction, transmission electron microscopy, atomic force microscopy, Raman spectroscopy, bulk electrical characterization, and many other techniques. Importantly, all of it will need assimilating and mutually reconciling to really begin to understand such complex materials; only then will we begin to picture the whole "elephant."

Acknowledgements This article is dedicated to the memory of Dr. Hirotake Okino, a great engineer, scientist, colleague and friend who died tragically in July 2006. Hirotake was the pioneer of new AFM-based techniques for investigation of the domain structures in ferroelectric materials and did much to illuminate the sub-micron scale structure of PMN $-x \mathrm{PT}$ and PZN $-x \mathrm{PT}$. He will be sadly missed.

The author acknowledges financial support from the Swiss National Science Foundation.

\section{References}

1. S.-E.E. Park, T.R. Shrout, J. Appl. Phys. 82, 1804-1811 (1997)

2. S.-E.E. Park, W. Hackenberger, Curr. Opin. Solid State Mater. Sci. 6, 11-18 (2002)
3. S.-E. Park, T.R. Shrout, IEEE Trans. Ultrason. Ferroelectr. Freq. Control 4, 1140-1147 (1997)

4. M.J. Zipparo, K.K. Shung, T.R. Shrout, IEEE Trans. Ultrason. Ferroelectr. Freq. Control 44, 1038-1048 (1997)

5. K.A. Snook, P.W. Rehrig, X. Jiang, R.J. Meyer, D. Markley, in Proceedings IEEE Ultrasonics Symposium, (2005), pp. 10651068

6. C.G. Oakley, M.J. Zipparo, in Proceedings of the 12th IEEE Ultrasonics Symposium, vol. 2 (2000), pp. 1157-1167

7. K. Harada, Y. Hosono, T. Kobayashi, Y. Yamashita, S. Wada, T. Tsurumi, J. Cryst. Growth 237, 848-852 (2002)

8. T. Ritter, X. Geng, K.K. Shung, P.D. Lopath, S.-E. Park, T.R. Shrout, IEEE Trans. Ultrason. Ferroelectr. Freq. Control 47, 792-800 (2000)

9. B. Noheda, Curr. Opin. Solid State Mater. Sci. 6, 27-34 (2002)

10. B. Noheda, D.E. Cox, Phase Transit. 79, 5-20 (2005)

11. H. Fu, R.E. Cohen, Nature 403, 281-283 (2000)

12. E.H. Kisi, R.O. Piltz, J.S. Forrester, C.J. Howard, J. Phys., Condens. Matter 15, 3631-3640 (2003)

13. K.K. Rajan, L.C. Lim, Appl. Phys. Lett. 83, 5277-5279 (2003)

14. M.K. Durbin, J.C. Hicks, S.-E. Park, T.R. Shrout, J. Appl. Phys. 87, 8159-8164 (2000)

15. K. Fujishiro, R. Vlokh, Y. Uesu, Y. Yamada, J.-M. Kiat, B. Dkhil, Y. Yamashita, Jpn. J. Appl. Phys. Pt. 1 37, 5246-5248 (1998)

16. L.C. Lim, F.J. Kumar, A. Amin, J. Appl. Phys. 93, 3671-3673 (2003)

17. J.-K. Lee, J.Y. Yi, K.-S. Hong, S.-E. Park, J. Millan, J. Appl. Phys. 91, 4474 (2002)

18. M. Budimir, D. Damjanovic, N. Setter, J. Appl. Phys. 94, 6753$6761(2003)$

19. J. Kuwata, K. Uchino, S. Nomura, Jpn. J. Appl. Phys. 21, 1298 $1302(1982)$

20. D. Damjanovic, M. Budimir, M. Davis, N. Setter, Appl. Phys. Lett. 83, 527-529 (2003)

21. R. Zhang, B. Jiang, W. Cao, Appl. Phys. Lett. 82, 3737-3739 (2003)

22. M. Davis, Thesis, Ecole Polytechnique Fédérale de Lausanne (EPFL), 2006

23. A.G. Khachaturyan, S.M. Shapiro, S. Semenovskaya, Phys. Rev., B 43, 832-843 (1991)

24. Y.M. Jin, Y.U. Wang, A.G. Khachaturyan, J. Appl. Phys. 94, 3629-3640 (2003)

25. D. Viehland, J. Appl. Phys. 88, 4794 (2000)

26. Y.U. Wang, Phys. Rev., B 73, 014113 (2006)

27. D.L. Corker, A.M. Glazer, R.W. Whatmore, A. Stallard, F. Fauth, J. Phys., Condens. Matter 10, 6251-6269 (1998)

28. A.M. Glazer, P.A. Thomas, K.Z. Baba-Kishi, G.K.H. Pang, C.W. Tai, Phys. Rev., B 70, 184123 (2004)

29. B. Noheda, J.A. Gonzalo, L.E. Cross, R. Guo, S.-E. Park, D.E. Cox, G. Shirane, Phys. Rev., B 61, 8687-8695 (2000)

30. A.J. Bell, J. Mater. Sci. 41, 13-25 (2006)

31. A.K. Singh, D. Pandey, O. Zaharko, Phys. Rev., B 74, 024101 (2006)

32. L.E. Cross, Ferroelectrics 76, 241-267 (1987)

33. D.-H. Lee, N.-K. Kim, Mater. Lett. 34, 299-304 (1998)

34. O. Noblanc, P. Gaucher, G. Calvarin, J. Appl. Phys. 79, 42914297 (1996)

35. A.A. Bokov, H. Luo, Z.-G. Ye, Mater. Sci. Eng., B 120, 206-209 (2005)

36. H. Fan, L. Kong, L. Zhang, X. Yao, J. Mater. Sci. 34, 6143-6149 (1999)

37. S.-A. Seo, K.H. Noh, S.-I. Kwun, J. Korean Phys. Soc. 35, 496499 (1999)

38. J. Han, W. Cao, Phys. Rev., B 68, 134102 (2003)

39. B. Jaffe, W.R. Cook, H. Jaffe, Piezoelectric Ceramics (Academic, 1971) 
40. C.-S. Tu, C.-L. Tsai, V.H. Schmidt, H. Luo, Z. Yin, J. Appl. Phys. 89, 7908-7916 (2001)

41. A. Lebon, H. Dammak, G. Calvarin, I.O. Ahmedou, J. Phys., Condens. Matter 14, 7035-7043 (2002)

42. H. Wang, H. Xu, H. Luo, Z. Yin, Appl. Phys. Lett. 87, 012904 (2005)

43. N.d. Mathan, E. Husson, G. Calvarin, J.R. Gavarri, A.W. Hewat, A. Morell, J. Phys., Condens. Matter 3, 8159-8171 (1991)

44. P.M. Gehring, W. Chen, Z.-G. Ye, G. Shirane, J. Phys., Condens. Matter 16, 7113-7121 (2004)

45. Z.-G. Ye, Y. Bing, J. Gao, A.A. Bokov, P. Stephens, B. Noheda, G. Shirane, Phys. Rev., B 67, 104104 (2003)

46. G. Xu, Z. Zhong, Y. Bing, Z.-G. Ye, C. Stock, G. Shirane, Phys. Rev., B 70, 064107 (2004)

47. K. Ohwada, K. Hirota, P.W. Rehrig, Y. Fujii, G. Shirane, Phys. Rev., B 67, 094111 (2003)

48. B. Dkhil, J.M. Kiat, G. Calvarin, G. Baldinozzi, S.B. Vakhrushev, E. Suard, Phys. Rev., B 65, 024104 (2001)

49. E.H. Kisi, J.S. Forrester, J. Phys., Condens. Matter 17, L381L384 (2005)

50. G. Xu, H. Hiraka, G. Shirane, K. Ohwada, Appl. Phys. Lett. 84, 3975-3977 (2004)

51. M. Davis, D. Damjanovic, N. Setter, J. Appl. Phys. 96, 28112815 (2004)

52. K.K. Rajan, M.J. Zhang, L.-C. Lim, Jpn. J. Appl. Phys. 44, 264 $266(2005)$

53. H. Cao, J. Li, D. Viehland, G. Xu, G. Shirane, Appl. Phys. Lett. 88, 072915 (2006)

54. H. Cao, J. Li, D. Viehland, G. Xu, Phys. Rev., B 73, 184110 (2006)

55. M. Matsushita, Y. Tachi, K. Echizenya, J. Cryst. Growth 237, 853-857 (2002)

56. H. Luo, G. Xu, H. Xu, P. Wang, Z. Yin, Jpn. J. Appl. Phys. Pt. 1 39, 5581-5585 (2000)

57. F.J. Kumar, L.-C. Lim, S.P. Lim, K.H. Lee, IEEE Trans. Ultrason. Ferroelectr. Freq. Control 50, 203-209 (2003)

58. P. Bao, F. Yan, X. Lu, J. Zhu, H. Shen, Y. Wang, H. Luo, Appl. Phys. Lett. 88, 092905 (2006)

59. A.A. Bokov, Z.-G. Ye, J. Appl. Phys. 91, 6656-6661 (2002)

60. R.R. Chien, V.H. Schmidt, L.-W. Hung, C.-S. Tu, J. Appl. Phys. 97, 114112 (2005)

61. D. Zekria, V.A. Shuvaeva, A.M. Glazer, J. Phys., Condens. Matter 17, 1593-1600 (2005)

62. Z.-G. Ye, M. Dong, J. Appl. Phys. 87, 2312-2319 (2000)

63. D.-L. Li, G.-S. Xu, P.-C. Wang, X.-M. Pan, H.-S. Luo, Z.-W. Yin, Ferroelectrics 253, 31-38 (2001)

64. G. Xu, H. Luo, Z. Qi, H. Xu, Z. Yin, J. Mater. Res. 16, 932-937 (2001)

65. A.-E. Renault, H. Dammak, G. Calvarin, M.P. Thi, P. Gaucher, Jpn. J. Appl. Phys. Pt. 1 41, 3846-3850 (2002)

66. J. Han, W. Cao, Appl. Phys. Lett. 83, 2040-2042 (2003)

67. E.K.H. Salje, Phase Transitions in Ferroelastic and Co-elastic Crystals (Cambridge University Press, Cambridge, 1993)

68. D. Viehland, M.-C. Kim, Z. Xu, J.-F. Li, Appl. Phys. Lett. 67, 2471-2473 (1995)

69. F. Bai, J. Li, D. Viehland, Appl. Phys. Lett. 85, 2313-2315 (2004)

70. Z. Xu, M.-C. Kim, J.-F. Li, D. Viehland, Philos. Mag., A 74, 395-406 (1996)

71. M.L. Mulvihill, L.E. Cross, W. Cao, K. Uchino, J. Am. Ceram. Soc. 80, 1462-1468 (1997)

72. M. Abplanalp, D. Barosova, P. Bridenbaugh, J. Erhart, J. Fousek, P. Gunter, J. Nosek, M. Sulc, J. Appl. Phys. 91, 3797-3805 (2002)

73. M. Iwata, K. Katsuraya, S. Tachizaki, J. Hlinka, I. Suzuki, M. Maeda, N. Yasuda, Y. Ishibashi, Jpn. J. Appl. Phys. 43, 6812$6815(2004)$
74. M. Iwata, K. Katsuraya, I. Suzuki, M. Maeda, N. Yasuda, Y. Ishibashi, Jpn. J. Appl. Phys. Part 1 42, 6201-6204 (2003)

75. S. Madeswaran, S.V. Rajasekaran, R. Jayavel, S. Ganesamoorthy, G. Behr, Mater. Sci. Eng., B 120, 32-36 (2005)

76. M. Zgonik, R. Schlesser, I. Biaggio, E. Voit, J. Tscherry, P. Gunter, J. Appl. Phys. 74, 1287-1297 (1993)

77. I.K. Bdikin, V.V. Shvartsman, A.L. Kholkin, Appl. Phys. Lett. 83, 4232-4234 (2003)

78. D.J. Quesnel, Presented at the 2006 US navy workshop on acoustic transduction materials and devices, State College, PA, USA

79. G. Burns, F.H. Dacol, Solid State Commun. 48, 853-856 (1983)

80. G. Xu, Z. Zhong, Y. Bing, Z.-G. Ye, G. Shirane, Nature Materials 5, 134-140 (2006)

81. A. Naberezhnov, S. Vakhrushev, B. Dorner, D. Strauch, H. Moudden, Eur. Phys. J., B 11, 13-20 (1999)

82. K. Hirota, Z.-G. Ye, S. Wakimoto, P.M. Gehring, G. Shirane, Phys. Rev., B 65, 104105 (2002)

83. H. Hellwig, A. Sehirlioglu, D.A. Payne, P. Han, Phys. Rev., B 73, 094126 (2006)

84. D. La-Orauttapong, J. Toulouse, Z.-G. Ye, W. Chen, R. Erwin, J. L. Robertson, Phys. Rev., B 67, 134110 (2003)

85. G. Schmidt, Ferroelectrics 78, 199-206 (1988)

86. A.A. Bokov, Ferroelectrics 131, 49-55 (1992)

87. M.D. Glinchuk, Br. Ceram., Trans. 103, 76-82 (2004)

88. A.A. Bokov, Z.-G. Ye, J. Mater. Sci. 41, 31-52 (2006)

89. W. Kleemann, J. Mater. Sci. 41, 129-136 (2006)

90. R. Blinc, V.V. Laguta, B. Zalar, J. Banys, J. Mater. Sci. 41, 27 30 (2006)

91. B. Noheda, D.E. Cox, G. Shirane, R. Guo, B. Jones, L.E. Cross, Phys. Rev., B 63, 014103 (2000)

92. T. Yamamoto, Jpn. J. Appl. Phys. Part 1 35, 5104-5108 (1996)

93. S.-E. Park, T.R. Shrout, Mater. Res. Innov. 1, 20-25 (1997)

94. G. A. Rossetti, Jr., W. Zhang, A.G. Khachaturyan, Appl. Phys. Lett. 88, 072912 (2006)

95. J.Y. Li, R.C. Rogan, E. Ustündag, K. Bhattacharya, Nature Materials 4, 776-781 (2005)

96. J.F. Nye, Physical Properties of Crystals, 2nd edn. (Clarendon Press, Oxford, 1985)

97. X. Liu, E.F. McCandlish, L.E. McCandlish, K. Mikulka-Bolen, R. Ramesh, F. Cosandey, G. A. Rossetti, Jr., R.E. Riman, Langmuir 21, 3207-3212 (2005)

98. G.A. Smolenskii, A.I. Agranovskaya, Sov. Phys., Solid State, 1429 (1959)

99. S.W. Choi, T.R. Shrout, S.J. Jang, A.S. Bhalla, Mater. Lett. 8, 253-255 (1989)

100. K. Uchino, S. Nomura, L.E. Cross, S.J. Jang, R.E. Newnham, J. Appl. Phys. 51, 1142-1145 (1980)

101. S.J. Jang, K. Uchino, S. Nomura, L.E. Cross, Ferroelectrics 27 , 31-34 (1980)

102. S.L. Swartz, T.R. Shrout, Mater. Res. Bull. 17, 1245-1250 (1982)

103. D.J. Taylor, D. Damjanovic, A.S. Bhalla, Ferroelectrics 118, 143-155 (1991)

104. S.W. Choi, T.R. Shrout, S.J. Jang, A.S. Bhalla, Ferroelectrics 100, 29-38 (1989)

105. J. Kuwata, K. Uchino, S. Nomura, Ferroelectrics 37, 579-582 (1981)

106. T.R. Shrout, Z.P. Chang, N. Kim, S. Markgraf, Ferroelectr., Lett. 12, 63-69 (1990)

107. D. Damjanovic, J. Am. Ceram. Soc. 88, 2663-2676 (2005)

108. J. Erhart, Phase Transit. 77, 989-1074 (2004)

109. J. Fousek, V. Janovec, J. Appl. Phys. 40, 135-142 (1969)

110. S. Wada, S.-E. Park, L.E. Cross, T.R. Shrout, Ferroelectrics 221, 147-155 (1999)

111. D.-S. Paik, S.-E. Park, S. Wada, S.-F. Liu, T.R. Shrout, J. Appl. Phys. 85, 1080-1083 (1999) 
112. M.K. Durbin, E.W. Jacobs, J.C. Hicks, Appl. Phys. Lett. 74, 2848-2850 (1999)

113. B. Noheda, D.E. Cox, G. Shirane, J.A. Gonzalo, L.E. Cross, S.-E. Park, Appl. Phys. Lett. 74, 2059-2061 (1999)

114. D. Vanderbilt, M.H. Cohen, Phys. Rev., B 63, 094108 (2001)

115. R.E. Newnham, Properties of Materials (Oxford University Press, Oxford, 2005)

116. X.-h. Du, J. Zheng, U. Belegundu, K. Uchino, Appl. Phys. Lett. 72, 2421-2423 (1998)

117. R. Clarke, R.W. Whatmore, J. Cryst. Growth 33, 29-38 (1976)

118. R. Guo, L.E. Cross, S.-E. Park, B. Noheda, D.E. Cox, G. Shirane, Phys. Rev. Lett. 84, 5423-5426 (2000)

119. L. Bellaiche, A. Garcia, D. Vanderbilt, Phys. Rev., B 64, 060103 (R) (2001)

120. M.J. Haun, E. Furman, S.J. Jang, L.E. Cross, Ferroelectrics 99, 13-25 (1989)

121. M.E. Lines, A.M. Glass, Principles and Applications of Ferroelectrics and Related Materials (Clarendon Press, Oxford, 1977)

122. D.E. Cox, B. Noheda, G. Shirane, Y. Uesu, K. Fujishiro, Y. Yamada, Appl. Phys. Lett. 79, 400-402 (2001)

123. J.-M. Kiat, Y. Uesu, B. Dkhil, M. Matsuda, C. Malibert, G. Calvarin, Phys. Rev., B 65, 064106 (2002)

124. Z.-G. Ye, B. Noheda, M. Dong, D. Cox, G. Shirane, Phys. Rev., B 64, 184114 (2001)

125. D. La-Orauttapong, B. Noheda, Z.-G. Ye, P.M. Gehring, J. Toulouse, D.E. Cox, G. Shirane, Phys. Rev., B 65, 144101 (2002)

126. B. Noheda, D.E. Cox, G. Shirane, J. Gao, Z.-G. Ye, Phys. Rev., B 66, 054104 (2002)

127. A.K. Singh, D. Pandey, Phys. Rev., B 67, 064102 (2003)

128. A.A. Bokov, Y.-G. Ye, Phys. Rev., B 66, 094112 (2002)

129. A.K. Singh, D. Pandey, Phys. Rev., B 68, 172103 (2003)

130. B. Noheda, D.E. Cox, G. Shirane, S.-E. Park, L.E. Cross, Z. Zhong, Phys. Rev. Lett. 86, 3891 (2001)

131. B. Noheda, Z. Zhong, D.E. Cox, G. Shirane, S.-E. Park, P. Rehrig, Phys. Rev., B 65, 224101 (2002)

132. K. Ohwada, K. Hirota, P.W. Rehrig, P.M. Gehring, B. Noheda, Y. Fujii, S.-E. Park, G. Shirane, J. Phys. Soc. Jpn. 70, 2778-2783 (2001)

133. B. Noheda, D.E. Cox, Phase Transit. 79, 5-20 (2006)

134. J.S. Forrester, R.O. Piltz, E.H. Kisi, G.J. McIntyre, J. Phys., Condens. Matter 13, L825-L833 (2001)

135. H. Cao, F. Bai, N. Wang, J. Li, D. Viehland, G. Xu, G. Shirane, Phys. Rev., B 72, 064104 (2005)

136. H. Cao, F. Bai, J. Li, D. Viehland, G. Xu, H. Hiraka, G. Shirane, J. Appl. Phys. 97, 094101 (2005)

137. F. Bai, N. Wang, J. Li, D. Viehland, P.M. Gehring, G. Xu, G. Shirane, J. Appl. Phys. 96, 1620-1627 (2004)

138. Y. Uesu, M. Matsuda, Y. Yamada, K. Fujishiro, D.E. Cox, B. Noheda, G. Shirane, J. Phys. Soc. Jpn. 71, 960-965 (2002)

139. A.A. Bokov, Z.-G. Ye, J. Appl. Phys. 95, 6347-6359 (2004)

140. G. Xu, H. Luo, H. Xu, Y. Yin, Phys. Rev., B 64, 020102(R) (2001)

141. V.A. Shuvaeva, A.M. Glazer, D. Zekria, J. Phys., Condens. Matter 17, 5709-5723 (2005)

142. M. Shen, D. Yao, W. Cao, Mater. Lett. 59, 3276-3279 (2005)

143. Y. Lu, D.-Y. Jeong, Z.-Y. Cheng, Q.M. Zhang, H.-S. Luo, Z.-W. Yin, D. Viehland, Appl. Phys. Lett. 78, 3109-3111 (2001)

144. C.-S. Tu, R.R. Chien, F.-T. Wang, V.H. Schmidt, P. Han, Phys. Rev., B 70, 220103(R) (2004)

145. Y. Lu, D.-Y. Jeong, Z.-Y. Cheng, T. Shrout, Q.M. Zhang, Appl. Phys. Lett. 80, 1918-1920 (2002)

146. S. Priya, J. Ryu, L.E. Cross, K. Uchino, D. Viehland, Ferroelectrics 274, 121-126 (2002)

147. A. Sehirlioglu, D.A. Payne, P. Han, J. Appl. Phys. 99, 064101 (2006)
148. Y. Guo, H. Luo, D. Ling, H. Xu, T. He, Z. Yin, J. Phys., Condens. Matter 15, L77-L82 (2003)

149. D.I. Woodward, J. Knudsen, I.M. Reaney, Phys. Rev., B 72, 104110 (2005)

150. M. Davis, D. Damjanovic, N. Setter, J. Appl. Phys. 96, (2004)

151. M. Davis, D. Damjanovic, N. Setter, J. Appl. Phys. 97, 064101 (2005)

152. D. Viehland, J.F. Li, J. Appl. Phys. 92, 7690-7692 (2002)

153. W. Ren, S.-F. Liu, B.K. Mukherjee, Appl. Phys. Lett. 80, 3174 $3176(2002)$

154. M. Davis, D. Damjanovic, N. Setter, Phys. Rev., B 73, 014115 (2006)

155. R.R. Chien, V.H. Schmidt, C.-S. Tu, L.-W. Hung, H. Luo, Phys. Rev., B 69, 172101 (2004)

156. C.-S. Tu, I.-C. Shih, V.H. Schmidt, R. Chien, Appl. Phys. Lett. 83, 1833-1835 (2003)

157. C.-S. Tu, H. Schmidt, I.-C. Shih, R. Chien, Phys. Rev., B 67, 020102(R) (2003)

158. E.A. McLaughlin, T. Liu, C.S. Lynch, Acta Mater. 52, 3849 3857 (2004)

159. Z. Feng, D. Lin, H. Luo, S. Li, D. Fang, J. Appl. Phys. 97, 024103 (2005)

160. Q. Wan, C. Chen, Y.P. Shen, J. Appl. Phys. 98, 024103 (2005)

161. A.J. Bell, J. Appl. Phys. 89, 3907-3914 (2001)

162. I.A. Sergienko, Y.M. Gufan, S. Urazhdin, Phys. Rev., B 65, 144104 (2002)

163. E. Dul'kin, M. Roth, P.-E. Janolin, B. Dkhil, Phys. Rev., B 73, $012102(2006)$

164. L.E. Cross, P. Hana, in Proceedings of 9th US-Japan Seminar on Dielectric and Piezoelectric Ceramics, 1999

165. A. Amin, M.J. Haun, B. Badger, H. McKinstry, L.E. Cross, Ferroelectrics 65, 107-130 (1985)

166. A. Amin, in Proceedings of 14th IEEE International Symposium on Applications of Ferroelectrics, 2004

167. H. Dammak, A.-E. Renault, P. Gaucher, M.P. Thi, G. Calvarin, Jpn. J. Appl. Phys. Pt. 1 10, 6477-6482 (2003)

168. R. Zhang, W. Cao, Appl. Phys. Lett. 85, 6380-6382 (2004)

169. R. Zhang, B. Jiang, W. Cao, Appl. Phys. Lett. 82, 787-789 (2003)

170. K.K. Rajan, J. Jin, L.C. Lim, Presented at the 2006 US navy workshop on acoustic transduction materials and devices, State College, PA, USA

171. M. Zgonik, P. Bernasconi, M. Duelli, R. Schlesser, P. Günter, M. H. Garrett, D. Rytz, Y. Zhu, X. Wu, Phys. Rev., B 50, 59415949 (1994)

172. M.J. Haun, E. Furman, S.J. Jang, L.E. Cross, IEEE Trans. Ultrason. Ferroelectr. Freq. Control 36, 393-401 (1989)

173. D. Damjanovic, M. Budimir, M. Davis, N. Setter, J. Mater. Sci. 41, 65-76 (2006)

174. M. Budimir, D. Damjanovic, N. Setter, Phys. Rev., B 73, 174106 (2006)

175. M.J. Haun, E. Furman, S.J. Jang, L.E. Cross, Ferroelectrics 99, 63-86 (1989)

176. R.R. Neurgaonkar, W.K. Cory, J. Opt. Am. B 3, 274-282 (1986)

177. T.R. Shrout, H. Chen, L.E. Cross, Ferroelectrics 74, 317-324 (1987)

178. Z. Li, M. Grimsditch, X. Xu, S.-K. Chan, Ferroelectrics 141, 313-325 (1993)

179. Z.-G. Ye, Curr. Opin. Solid State Mater. Sci. 6, 35-44 (2002)

180. M. Iwata, T. Araki, M. Maeda, I. Suzuki, H. Ohwa, N. Yasuda, H. Orihara, Y. Ishibashi, Jpn. J. Appl. Phys. Pt. 1 11B, 7003-7006 (2002)

181. V.Y. Topolov, A.V. Turik, Defect Diffus. Forum 123-124, 31-50 (1995)

182. G. Metrat, Ferroelectrics 26, 801-804 (1980)

183. V.Y. Topolov, Z.-G. Ye, Phys. Rev., B 70, 094113 (2004) 
184. V.Y. Topolov, J. Phys., Condens. Matter 16, 1-7 (2004)

185. V.Y. Topolov, A.V. Turik, J. Phys., Condens. Matter 13, L771L775 (2001)

186. J. Yin, W. Cao, J. Appl. Phys. 87, 7438-7441 (2000)

187. J. Yin, W. Cao, J. Appl. Phys. 92, 444-448 (2002)

188. J. Erhart, W. Cao, J. Appl. Phys. 86, 1073-1081 (1999)

189. M. Shanthi, K.H. Hoe, C.Y.H. Lim, L.C. Lim, Appl. Phys. Lett. 86, 262908 (2005)

190. Y. Guo, H. Luo, K. Chen, H. Xu, X. Zhang, Z. Yin, J. Appl. Phys. 92, 6134-6138 (2002)

191. A.-E. Renault, H. Dammak, G. Calvarin, P. Gaucher, M.P. Thi, J. Appl. Phys. 97, 044105 (2005)

192. J.H. Ro, M. Cha, Appl. Phys. Lett. 77, 15 (2000), (August)

193. V.D. Kugel, G. Rosenman, Appl. Phys. Lett. 65, 2398-2400 (1994)

194. Y. Zhang, I.S. Baturin, E. Aulbach, D.C. Lupascu, A.L. Kholkin, V.Y. Shur, J. Rödel, Appl. Phys. Lett. 86, 012910 (2005)

195. M. Shen, W. Cao, Appl. Phys. Lett. 86, 192909 (2005)

196. M. Ozgul, S. Trolier-McKinstry, C.A. Randall, J. Appl. Phys. 95, 4296-4302 (2004)

197. W. Yhu, L.E. Cross, Appl. Phys. Lett. 84, 2388-2390 (2004)

198. G. Xu, P.M. Gehring, G. Shirane, Phys. Rev., B 72, 214106 (2005)

199. A. Sehirlioglu, D.A. Payne, Phys. Rev., B 72, 214110 (2005)

200. E.K.H. Salje, Acta Crystallogr., A 47, 453-469 (1991)

201. A.D. Hilton, C.A. Randall, D.J. Barber, T.R. Shrout, Ferroelectrics 93, 379-386 (1989)

202. D.A. Porter, K.E. Easterling, Phase Transformations in Metals and Alloys, 2nd edn. (Chapman and Hall, London, 1992)

203. G. Schmidt, G. Borchhardt, J.V. Cieminksi, D. Grützmann, E. Purinsch, V.A. Isupov, Ferroelectrics 42, 3-9 (1982)

204. G. Burns, F.H. Dacol, Solid State Commun. 42, 9-12 (1982)

205. D. Viehland, J. Li, E.V. Colla, J. Appl. Phys. 96, 3379-3381 (2004)

206. X. Meng, K. Baba-Kishi, H. Chan, C.-1. Choy, H. Luo, Jpn. J. Appl. Phys. Part 1 41, 2999-3005 (2002)

207. E. Salje, K. Parlinski, Supercond. Sci. Technol. 4, $93-97$ (1991)

208. K. Parlinski, V. Heine, E.K.H. Salje, J. Phys., Condens. Matter 5, 497-518 (1993)

209. J. Sapriel, Phys. Rev., B 12, 5128-5140 (1975)

210. M. Ozgul, K. Takemura, S. Trolier-McKinstry, C.A. Randall, J. Appl. Phys. 89, 5100-5106 (2001)

211. M. Davis, D. Damjanovic, N. Setter, J. Appl. Phys. (submitted), (2006)

212. D. Damjanovic, in Science in Hysteresis, vol. 3, ed. by G. Bertotti. Hysteresis in Piezoelectric and Ferroelectric Materials (Elsevier, 2005), p. 337

213. M. Davis, D. Damjanovic, N. Setter, J. Appl. Phys. 95, 5679$5684(2004)$

214. M. Davis, D. Damjanovic, N. Setter, J. Appl. Phys. 100, 084103 (2006)

215. H. Wang, J. Zhu, N. Lu, A.A. Bokov, Z.-G. Ye, X.W. Zhang, Appl. Phys. Lett. 89, 042908 (2006)
216. D. Viehland, J.F. Li, J. Appl. Phys. 92, 3985-3989 (2002)

217. D. Viehland, A. Amin, J.F. Li, Appl. Phys. Lett. 79, 1006-1008 (2001)

218. J. Frantti, S. Eriksson, S. Hull, V. Lantto, H. Rundlöf, M. Kakihana, J. Phys., Condens. Matter 15, 6031-6041 (2003)

219. G. Burns, F.H. Dacol, J.P. Remeika, W. Taylor, Phys. Rev., B 26, 2707-2709 (1982)

220. C. Kittel, Introduction to Solid State Physics, 7th edn. (Wiley, New York, 1996)

221. R. Comés, M. Lambert, A. Guinier, Acta Crystallogr., A 26, 244-254 (1969)

222. R. Comès, M. Lambert, A. Guinier, Solid State Commun. 6, 715-719 (1968)

223. P.J. Edwardson, Phys. Rev. Lett. 63, 55-58 (1989)

224. S. Wada, T. Suzuki, M. Osada, M. Kakihana, T. Noma, Jpn. J. Appl. Phys. Part 1 37, 5385-5393 (1998)

225. W. Zhong, D. Vanderbilt, K.M. Rabe, Phys. Rev. Lett. 73, 18611864 (1994)

226. A. Kania, E. Talik, M. Kruczek, A. Slodczyk, J. Phys., Condens. Matter 17, 6737-6749 (2005)

227. I. Grinberg, V.R. Cooper, A.M. Rappe, Nature 419, 909-911 (2002)

228. R. Haumont, A. Al-Barakaty, B. Dkhil, J.M. Kiat, L. Bellaiche, Phys. Rev., B 71, 104106 (2005)

229. P.K. Davies, M.A. Akbas, J. Phys. Chem. Solids 61, 159-166 (2000)

230. B.P. Burton, E. Cockayne, U.V. Waghmare, Phys. Rev., B 72, 064113 (2005)

231. L. Bellaiche, A. Garcia, D. Vanderbilt, Phys. Rev. Lett. 84, 5427-5430 (2000)

232. M. Davis, D. Damjanovic, D. Hayem, N. Setter, J. Appl. Phys. 98, $014102(2005)$

233. S. Wada, K. Muraoka, H. Kakemoto, T. Tsurumi, H. Kumagai, Jpn. J. Appl. Phys. Part 1 43, 6692-6700 (2004)

234. S. Wada, K. Yako, H. Kakemoto, J. Erhart, T. Tsurumi, Key Eng. Mater. 269, 19-22 (2004)

235. S. Wada, H. Kakemoto, T. Tsurumi, Mater. Trans. 45, 178-187 (2004)

236. S. Wada, K. Yako, H. Kakemoto, T. Tsurumi, T. Kiguchi, J. Appl. Phys. 98, 014109 (2005)

237. K. Yako, H. Kakemoto, T. Tsurumi, S. Wada, Mater. Sci. Eng. B 120, 181-185 (2005)

238. R. Ahluwalia, T. Lookman, A. Saxena, W. Cao, Phys. Rev., B 72, 014112 (2005)

239. R. Ahluwalia, T. Lookman, A. Saxena, W. Cao, Appl. Phys. Lett. 84, 3450-3452 (2004)

240. M. Budimir, D. Damjanovic, N. Setter, Appl. Phys. Lett. 85, 2890-2892 (2004)

241. M. Ahart, A. Asthagiri, P. Dera, H.-k. Mao, R.E. Cohen, R.J. Hemley, Appl. Phys. Lett. 88, 042908 (2006)

242. T. Liu, C.S. Lynch, Acta Mater. 51, 407-416 (2003)

243. J. Yin, B. Jiang, W. Cao, IEEE Trans. Ultrason. Ferroelectr. Freq. Control 47, 285-291 (2000)

244. Z. Kutnjak, J. Petzelt, R. Blinc, Nature 441, 956-959 (2006) 